\title{
Oblique derivative problems and Feller semigroups with discontinuous coefficients
}

\author{
Kazuaki Taira \\ Dedicated to Professor Elmar Schrohe on the occasion of his 65th birthday
}

the date of receipt and acceptance should be inserted later

\begin{abstract}
This paper is devoted to the functional analytic approach to the problem of existence of Markov processes with an oblique derivative boundary condition for second-order, uniformly elliptic differential operators with discontinuous coefficients. More precisely, we construct Feller semigroups associated with absorption, reflection, drift and sticking (or viscosity) phenomena at the boundary. The approach here is distinguished by the extensive use of the ideas and techniques characteristic of the recent developments in the Calderón-Zygmund theory of singular integral operators with non-smooth kernels.
\end{abstract}

Keywords Feller semigroup · uniformly elliptic differential operator · VMO function $\cdot$ oblique derivative boundary condition $\cdot$ singular integral $\cdot$ maximum principle

Mathematics Subject Classification (2010) 47D07 - 35J25 - 47D05 - 60J35 . $60 \mathrm{~J} 60$

\section{Contents}

1 Introduction and Main Results ... . . . . . . . . . . . . . . . . . . . . . 2

2 The Spaces BMO and VMO . . . . . . . . . . . . . . . . . . . . 8

3 Generation Theorems for Feller Semigroups . . . . . . . . . . . . . . . . . . . 9

4 The Dirichlet Problem . . . . . . . . . . . . . . . . . . . . . . . . . 11

5 The Oblique Derivative Problem . . . . . . . . . . . . . . . . . . . . . 12

6 Proof of Theorem 1.2. . . . . . . . . . . . . . . . . . . . . . . . 17

7 Feller Semigroups and Boundary Value Problems . . . . . . . . . . . . . . . . . . . 21

8 Proof of Theorem 1.1. . . . . . . . . . . . . . . . . . . . 33

9 Proof of Remark 7.2 . . . . . . . . . . . . . . . . . . . . . . . . . . . . . 40

10 Concluding Remarks . . . . . . . . . . . . . . . . . . . . . . . . . . . 42

Kazuaki Taira

Institute of Mathematics, University of Tsukuba, Tsukuba 305-8571, Japan

E-mail: taira@math.tsukuba.ac.jp 


\section{Introduction and Main Results}

Let $\Omega$ be a bounded domain in Euclidean space $\mathbf{R}^{N}, N \geq 3$, with boundary $\partial \Omega$ of class $C^{1,1}$. We consider a second-order, elliptic differential operator $A$ with real discontinuous coefficients of the form

$$
A u:=\sum_{i, j=1}^{N} a^{i j}(x) \frac{\partial^{2} u}{\partial x_{i} \partial x_{j}}+\sum_{i=1}^{N} b^{i}(x) \frac{\partial u}{\partial x_{i}}+c(x) u .
$$

In the case of continuous coefficients $a^{i j}(x)$, an $L^{p}$ Schauder theory has been elaborated for second-order, uniformly elliptic differential operators (see [3], [17], [19]). However, the situation becomes rather difficult if we try to allow discontinuity on the $a^{i j}(x)$. In fact, it is known (see [26], [27], [38]) that arbitrary discontinuity of the $a^{i j}(x)$ breaks down as the $L^{p}$ Schauder theory, except for the two-dimensional case $(N=2)$. In order to handle with the multidimensional case $(N \geq 3)$, additional conditions on the $a^{i j}(x)$ should be required. Here we shall see that the relevant condition is that the coefficients $a^{i j}(x)$ belong to the Sarason class VMO of functions with vanishing mean oscillation. We recall that VMO consists of the John-Nirenberg class BMO of functions with bounded mean oscillation whose integral oscillation over balls shrinking to a point converge uniformly to zero (see Section 2).

Our approach here is distinguished by the extensive use of the CalderónZygmund theory of singular integrals (see [11]). Since second-order elliptic differential operators are pseudo-differential operators only if the coefficients are smooth, we cannot make use of the theory of pseudo-differential operators as in the previous work [35]. Singular integral operators provide a powerful tool to deal with smoothness of solutions of partial differential equations, with minimal assumptions of regularity on the coefficients. Several recent developments in the theory of singular integrals (see [12], [13], [31], [10], [14], [24], [25]) have made possible further progress in the study of elliptic boundary value problems with VMO coefficients and hence in the study of Markov processes.

It should be emphasized that the condition $N \geq 3$ is essential in the whole theory of singular integrals. In fact, we make use of integral representation formulas for the second derivatives of functions in the Sobolev spaces $W^{2, p}(\Omega)$ that are based on the fundamental solutions of elliptic differential operators with constant coefficients (see [12, Section 3], [25, Chapter 2]).

\subsection{Formulation of the Problem}

Throughout this paper, we assume that the coefficients $a^{i j}(x), b^{i}(x)$ and $c(x)$ of the differential operator $A$ satisfy the following three conditions (1), (2) and (3):

(1) $a^{i j}(x) \in \mathrm{VMO} \cap L^{\infty}(\Omega), a^{i j}(x)=a^{j i}(x)$ for almost all $x \in \Omega$ and there exist a constant $\lambda>0$ such that

$$
\frac{1}{\lambda}|\xi|^{2} \leq \sum_{i, j=1}^{N} a^{i j}(x) \xi_{i} \xi_{j} \leq \lambda|\xi|^{2} \quad \text { for almost all } x \in \Omega \text { and all } \xi \in \mathbf{R}^{N} .
$$

(2) $b^{i}(x) \in L^{\infty}(\Omega)$ for $1 \leq i \leq N$. 
(3) $c(x) \in L^{\infty}(\Omega)$ and $c(x) \leq 0$ for almost all $x \in \Omega$.

The differential operator $A$ is called a diffusion operator which describes analytically a strong Markov process with continuous paths in the interior $\Omega$ such as Brownian motion (see Figure 1.1 below). The differential opertor $A$ is a special case of Waldenfels integro-differential operators ([43], [35, Chapter 10]).

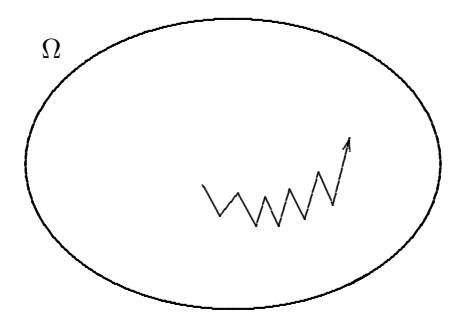

Fig. 1.1 A Markovian particle moves continuously

Moreover, we consider a first-order, boundary operator of the form

$$
\begin{aligned}
L u & :=\mu\left(x^{\prime}\right) \frac{\partial u}{\partial \mathbf{n}}+\beta\left(x^{\prime}\right) \cdot \partial_{x^{\prime}} u+\gamma\left(x^{\prime}\right) u-\delta\left(x^{\prime}\right)\left(\left.A u\right|_{\partial \Omega}\right) \\
& =\mu\left(x^{\prime}\right) \frac{\partial u}{\partial \mathbf{n}}+\sum_{j=1}^{N-1} \beta^{j}\left(x^{\prime}\right) \frac{\partial u}{\partial x_{j}}+\gamma\left(x^{\prime}\right) u-\delta\left(x^{\prime}\right)\left(\left.A u\right|_{\partial \Omega}\right) \quad \text { on } \partial \Omega .
\end{aligned}
$$

Here the local coordinate systems $\left(x^{\prime}, x_{N}\right)=\left(x_{1}, x_{2}, \ldots, x_{N-1}\right)$ give local coordinates near the boundary $\partial \Omega$ such that

$$
\left\{\begin{array}{l}
\Omega=\left\{\left(x^{\prime}, x_{N}\right): x_{N}>0\right\}, \\
\partial \Omega=\left\{\left(x^{\prime}, x_{N}\right): x_{N}=0\right\} .
\end{array}\right.
$$

We assume that the coefficients $\mu\left(x^{\prime}\right), \beta^{j}\left(x^{\prime}\right), \gamma\left(x^{\prime}\right)$ and $\delta\left(x^{\prime}\right)$ of the boundary operator $L$ satisfy the following four conditions (4), (5), (6) and (7) (see Figure $1.2)$ :

(4) $\mu\left(x^{\prime}\right)$ is a Lipschitz continuous function on $\partial \Omega$ and $\mu\left(x^{\prime}\right) \geq 0$ on $\partial \Omega$.

(5) $\beta^{j}\left(x^{\prime}\right)$ are Lipschitz continuous functions on $\partial \Omega$.

(6) $\gamma\left(x^{\prime}\right)$ is a Lipschitz continuous function on $\partial \Omega$ and $\gamma\left(x^{\prime}\right) \leq 0$ on $\partial \Omega$.

(7) $\delta\left(x^{\prime}\right)$ is a Lipschitz continuous function on $\partial \Omega$ and $\delta\left(x^{\prime}\right) \geq 0$ on $\partial \Omega$.

(8) $\mathbf{n}=\left(n_{1}, n_{2}, \ldots, n_{N}\right)$ is the unit inward normal to the boundary $\partial \Omega$.

The boundary condition $L$ is called a first-order, Wentzell (Ventcel') boundary condition (see [44], [35, Chapter 9]). The four terms of $L$

$$
\mu\left(x^{\prime}\right) \frac{\partial u}{\partial \mathbf{n}}, \quad \beta\left(x^{\prime}\right) \cdot \partial_{x^{\prime}} u, \quad \gamma\left(x^{\prime}\right) u, \quad \delta\left(x^{\prime}\right)\left(\left.A u\right|_{\partial \Omega}\right)
$$

are supposed to correspond to the reflection phenomenon, the drift phenomenon along the boundary, the absorption phenomenon and the sticking (or viscosity) phenomenon, respectively (see Figures 1.3 and 1.4 below). 


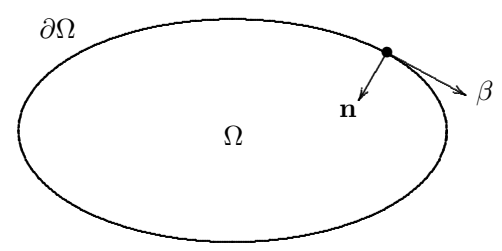

Fig. 1.2 The tangent vector field $\beta$ and the unit inward normal $\mathbf{n}$ to the boundary $\partial \Omega$

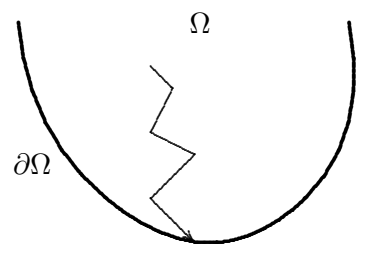

absorption

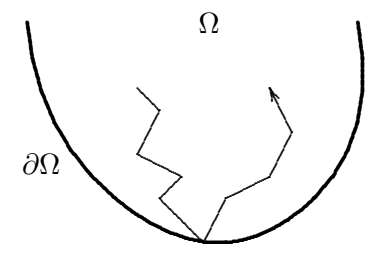

reflection

Fig. 1.3 The absorption phenomenon and the reflection phenomenon

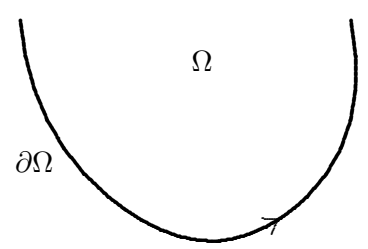

drift along the boundary

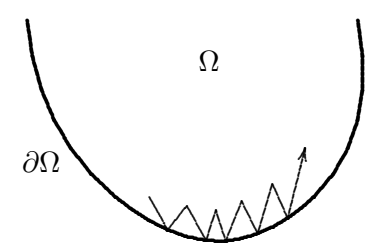

sticking (viscosity)

Fig. 1.4 The drift phenomenon along $\partial \Omega$ and the sticking phenomenon

Let $C(\bar{\Omega})$ be the Banach space of real-valued, continuous functions on the closure $\bar{\Omega}=\Omega \cup \partial \Omega$, equipped with the maximum norm

$$
\|f\|_{C(\bar{\Omega})}=\max _{x \in \bar{\Omega}}|f(x)| \quad \text { for } f \in C(\bar{\Omega}) .
$$

A strongly continuous semigroup $\left\{T_{t}\right\}_{t \geq 0}$ on the space $C(\bar{\Omega})$ is called a Feller semigroup on the state space $\bar{\Omega}$ if it is non-negative and contractive on the Banach space $C(\bar{\Omega})$, that is, if it satisfies the condition

$$
f \in C(\bar{\Omega}), 0 \leq f(x) \leq 1 \quad \text { on } \bar{\Omega} \Longrightarrow 0 \leq T_{t} f(x) \leq 1 \quad \text { on } \bar{\Omega} \text { for all } t \geq 0 .
$$

It is known (see [16], [35, p. 447, Theorem 9.34]) that if $T_{t}$ is a Feller semigroup on the Banach space $C(\bar{\Omega})$, then there exists a unique Markov transition function $p_{t}(x, \cdot)$ on the state space $\bar{\Omega}$ such that

$$
T_{t} f(x)=\int_{\bar{\Omega}} p_{t}(x, d y) f(y) \quad \text { for } f \in C(\bar{\Omega}) .
$$

Furthermore, it can be shown (see [15], [35, p. 439, Theorem 9.28]) that the function $p_{t}(x, \cdot)$ is the transition function of some strong Markov process whose paths are right-continuous and have no discontinuities other than jumps; hence the value 
$p_{t}(x, E)$ expresses the transition probability that a Markovian particle starting at position $x$ will be found in the set $E$ at time $t$.

The purpose of this paper is devoted to the functional analytic approach to the problem of existence of Markov processes in probability theory. More precisely, we consider the following problem (see [35, p. 12, Problem 1.1]):

Problem 1 Conversely, given analytic data $(A, L)$, can we construct a Feller semigroup $\left\{T_{t}\right\}_{t \geq 0}$ whose infinitesimal generator $\mathfrak{A}$ is characterized by $(A, L)$ ?

\subsection{Statement of Main Results}

The next theorem asserts that there exists a Feller semigroup corresponding to such a diffusion phenomenon that a Markovian particle moves continuously in the state space, with absorption, reflection, drift and sticking phenomena at the boundary (cf. [8, Théorème XIX]):

Theorem 1.1 Let $N \geq 3$. If $N<p<\infty$, we define a linear operator $\mathfrak{A}$ from $C(\bar{\Omega})$ into itself as follows (see Remark 8.1 in Section 8):

(a) The domain $D(\mathfrak{A})$ is the set

$$
D(\mathfrak{A})=\left\{u \in W^{2, p}(\Omega): A u \in C(\bar{\Omega}), L u=0 \text { on } \partial \Omega\right\} .
$$

(b) $\mathfrak{A} u=A u$ for every $u \in D(\mathfrak{A})$.

Here $A u$ and $L u$ are taken in the sense of distributions.

Assume that the following condition is satisfied:

(H.1) $\mu\left(x^{\prime}\right)>0$ on $\partial \Omega$.

Then the operator $\mathfrak{A}$ is the infinitesimal generator of a Feller semigroup on the state space $\bar{\Omega}$.

Rephrased, Theorem 1.1 asserts that there exists a Feller semigroup corresponding to such a diffusion phenomenon that a Markovian particle moves continuously in the state space with absorption, reflection, drift and sticking phenomena at the boundary (see Figure 1.5).

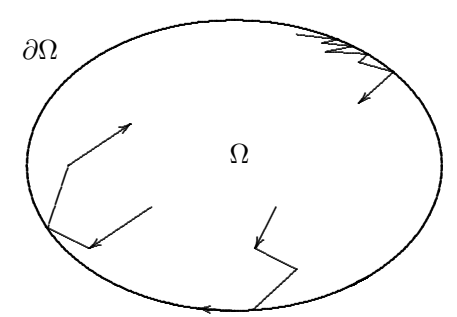

Fig. 1.5 A probabilistic meaning of Theorem 1.1 with absorption, reflection, drift and sticking phenomena at the boundary 
The most crucial point in the proof of Theorem 1.1 is how to treat the term $\delta\left(x^{\prime}\right)\left(\left.A u\right|_{\partial \Omega}\right)$ of sticking phenomenon in the first-order Wentzell boundary condition

$$
L u=\mu\left(x^{\prime}\right) \frac{\partial u}{\partial \mathbf{n}}+\beta\left(x^{\prime}\right) \cdot \partial_{x^{\prime}} u+\gamma\left(x^{\prime}\right) u-\delta\left(x^{\prime}\right)\left(\left.A u\right|_{\partial \Omega}\right) \quad \text { on } \partial \Omega
$$

as a term of perturbation of the oblique derivative boundary condition $\left(\delta\left(x^{\prime}\right) \equiv 0\right)$

$$
L_{\nu} u:=\mu\left(x^{\prime}\right) \frac{\partial u}{\partial \mathbf{n}}+\beta\left(x^{\prime}\right) \cdot \partial_{x^{\prime}} u+\gamma\left(x^{\prime}\right) u \quad \text { on } \partial \Omega .
$$

To do this, in Section 6 we prove the following generation theorem for Feller semigroups with oblique derivative boundary condition:

Theorem 1.2 Let $N \geq 3$. If $N<p<\infty$, we define a linear operator $\mathfrak{A}_{\nu}$ from $C(\bar{\Omega})$ into itself as follows:

(c) The domain $D\left(\mathfrak{A}_{\nu}\right)$ is the set

$$
D\left(\mathfrak{A}_{\nu}\right)=\left\{u \in W^{2, p}(\Omega): A u \in C(\bar{\Omega}), L_{\nu} u=0 \text { on } \partial \Omega\right\} .
$$

(d) $\mathfrak{A}_{\nu} u=A u$ for every $u \in D\left(\mathfrak{A}_{\nu}\right)$.

Here $A u$ and $L_{\nu} u$ are taken in the sense of distributions.

If condition (H.1) is satisfied, then the operator $\mathfrak{A}_{\nu}$ is the infinitesimal generator of a Feller semigroup on the state space $\bar{\Omega}$.

Rephrased, Theorem 1.2 asserts that there exists a Feller semigroup corresponding to such a diffusion phenomenon that a Markovian particle moves continuously in the state space with absorption, reflection and drift phenomena at the boundary (see Figure 1.6).

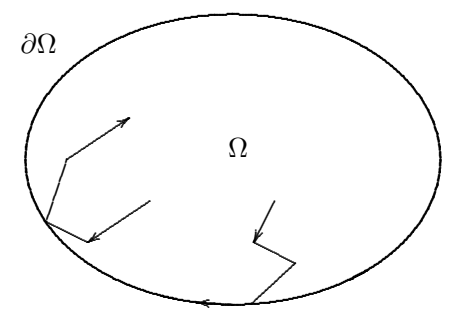

Fig. 1.6 A probabilistic meaning of Theorem 1.2 with absorption, reflection and drift phenomena at the boundary

In the previous paper [34, Theorems 1.1 and 1.2], we constructed Feller semigroups for second-order, uniformly elliptic differential operators with $V M O$ coefficients under the additional condition that

(H.2) $\gamma\left(x^{\prime}\right)<0$ on $\partial \Omega$.

This implies that the boundary condition $L_{\nu}$ is not the pure oblique derivative boundary condition.

Therefore, Theorems 1.1 and 1.2 substantially extend the main results of [34] (see Table 10.1 in the last Section 10). 


\subsection{Summary of the Contents}

The rest of this paper is organized as follows.

In Section 2 we summarize some important topics from real analysis such as BMO and VMO functions (Proposition 2.1). In Section 3 we provide a brief description of the basic definitions and results about Feller semigroups associated with Markov processes in probability theory, which forms a functional analytic background for the proof Theorems 1.1 and 1.2. In particular, we formulate the Hille-Yosida theorem adapted to the present context (Theorem 3.1 and Corollary 3.1). Moreover, we give two useful criteria in order that a linear operator is the infinitesimal generator of some Feller semigroup (Theorem 3.2 and Corollary 3.2). These two sections are intended as a brief introduction to our problem and results in such a fashion that a broad spectrum of readers could understand.

In Section 4 we consider the Dirichlet problem for the diffusion operator $A$ with VMO coefficients in the framework of Sobolev spaces of $L^{p}$ type, and state an existence and uniqueness theorem for the non-homogeneous Dirichlet problem (Theorem 4.1).

In Section 5 we study the oblique derivative problem in the framework of Sobolev spaces of $L^{p}$ type, and prove an existence and uniqueness theorem for the oblique derivative problem with VMO coefficients under condition (H.1) (Theorem 5.1). The main purpose of this section is to drop the additional condition (H.2) on the boundary condition $L_{\nu}$. In fact, by using the distance function

$$
d(x)=\operatorname{dist}(x, \partial \Omega) \text { for } x \in \bar{\Omega},
$$

we show that Theorem 5.1 under condition (H.1) may be reduced to [34, Theorem 4.1] (see Subsection 5.2). The uniqueness result (Theorem 5.1) follows from a variant of the Bakel'man-Aleksandrov maximum principle in the framework of Sobolev spaces due to Lieberman [22] (Theorem 5.3).

Section 6 is devoted to the proof of Theorem 1.2 under condition (H.1). By using Theorem 5.1, we verify all the conditions in the Hille-Yosida theorem (Theorem 3.1) for the closed operator $\mathfrak{A}_{\nu}$ defined by formula (1.6).

Section 7 is the heart of the subject. Its purpose is to prove a general existence theorem for Feller semigroups with first-order Wentzell boundary condition in terms of elliptic boundary value problems (Theorem 7.2), which is based on the main idea of Taira [32]. Intuitively, Theorem 7.2 asserts that we can "piece together" a strong Markov process on the boundary $\partial \Omega$ with $A$-diffusion in the interior $\Omega$ to construct a strong Markov process on the closure $\bar{\Omega}=\Omega \cup \partial \Omega$.

In Section 8 we prove Theorem 1.1 under condition (H.1). In fact, we make use of the generation theorem for Feller semigroups with oblique derivative boundary condition $L_{\nu}$ (Theorem 1.2) to verify all the conditions of a version of the HilleYosida-Ray theorem (Theorem 3.2) for the closed operator $\mathfrak{A}$ defined by formula (1.4). The proof of Theorem 1.1 is carried out in a series of propositions (Propositions 8.1, 8.2 and 8.3). In the proof we make good use of the Boutet de Monvel calculus [9] just as in [35, Section 7.7] (see Figure 8.1).

In Section 9 we prove Remark 7.2, which gives a precise definition of the definition (1.4) (and the definition (1.6)) in terms of the closed operators $\bar{A}$ and $\overline{L H_{\alpha}}$.

In the last Section 10, we give an overview of the classical Schauder theory versus the Calderón-Zygmund theory of singular integrals for general results on generation theorems for Feller semigroups (see Table 10.1). 


\section{The Spaces BMO and VMO}

In this section we recall some basic definitions and results concerning BMO and VMO functions on $\mathbf{R}^{N}$. For more thorough treatments of this subject, the reader might be referred to Garnett [18] and Torchinsky [39].

A function $f(x) \in L_{\text {loc }}^{1}\left(\mathbf{R}^{N}\right)$ is said to be of bounded mean oscillation, $f(x) \in$ $\mathrm{BMO}$, if it satisfies the condition (see [21])

$$
\|f\|_{*}:=\sup _{B} \frac{1}{|B|} \int_{B}\left|f(x)-f_{B}\right| d x<\infty
$$

where the supremum is taken over all balls $B$ in $\mathbf{R}^{N}$ and $f_{B}$ is the average of $f$ over $B$

$$
f_{B}:=\frac{1}{|B|} \int_{B} f(x) d x .
$$

It should be noticed that the quantity $\|f\|_{*}$ defines a norm on the quotient space $\mathrm{BMO} / \mathbf{R}$.

Next we introduce a subspace of BMO functions whose BMO norm over a ball vanishes as the radius of the ball tends to zero. More precisely, if $f(x) \in \mathrm{BMO}$ and $r>0$, then we let

$$
\eta(r):=\sup _{\rho \leq r} \frac{1}{|B|} \int_{B}\left|f(x)-f_{B}\right| d x
$$

where the supremum is taken over all balls $B$ with radius $\rho \leq r$.

A function $f(x) \in \mathrm{BMO}$ has vanishing mean oscillation, $f(x) \in \mathrm{VMO}$, if it satisfies the condition (see [28])

$$
\lim _{r \downarrow 0} \eta(r)=0
$$

The function $\eta(r)$ is called the VMO modulus of $f$.

The assumption $a^{i j}(x) \in \mathrm{VMO}$ means a kind of continuity in the average sense, not in the pointwise sense. This property implies that VMO functions may be approximated by smooth functions.

The relationship between BMO and its subspace VMO is quite similar to the relationship between $L^{\infty}$ and its subspace BUC of bounded uniformly continuous functions (see Figure 2.1).

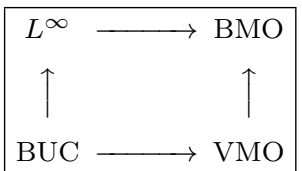

Fig. 2.1 The spaces $L^{\infty}, \mathrm{BMO}, \mathrm{BUC}$ and $\mathrm{VMO}$

The next proposition collects some important results concerning VMO functions (see [18, Chapter VI, Theorem 5.1], [39, Chapter VIII]): 
Proposition 2.1 (i) If $f(x) \in \mathrm{VMO}$, then, for any $\varepsilon>0$ there exists a uniformly continuous function $g_{\varepsilon}(x)$ on $\mathbf{R}^{N}$ such that $\left\|f-g_{\varepsilon}\right\|_{*}<\varepsilon$.

(ii) Uniformly continuous functions that belong to BMO are VMO functions.

(iii) VMO is a closed subspace of BMO. 1.3]).

(iv) VMO functions are invariant under $C^{1,1}$-diffeomorphisms ([1, Proposition

\section{Generation Theorems for Feller Semigroups}

This section is devoted to the Hille-Yosida theory of Feller semigroups.

Let $K$ be a locally compact, separable metric space and let $C(K)$ be the space of real-valued, bounded continuous functions $f$ on $K$. The space $C(K)$ is a Banach space with the supremum norm

$$
\|f\|=\sup _{x \in K}|f(x)|
$$

We add a point $\partial$ to the space $K$ as the point at infinity if $K$ is not compact, and as an isolated point if $K$ is compact. Thus the space $K_{\partial}=K \cup\{\partial\}$ is compact. Then we introduce a closed subspace of $C(K)$ as follows:

$$
C_{0}(K)=\left\{f \in C(K): \lim _{x \rightarrow \partial} f(x)=0\right\} .
$$

It should be noticed that the space $C_{0}(K)$ may be identified with the subspace of $C\left(K_{\partial}\right)$ which consists of all functions $f$ satisfying the condition $f(\partial)=0$ :

$$
C_{0}(K)=\left\{f \in C\left(K_{\partial}\right): f(\partial)=0\right\} .
$$

We remark that $C_{0}(K)$ may be identified with $C(K)$ if $K$ is compact.

A family $\left\{T_{t}\right\}_{t \geq 0}$ of bounded linear operators acting on the Banach space $C_{0}(K)$ is called a Feller semigroup on the state space $K$ if it satisfies the following three conditions (i), (ii) and (iii):

(i) $T_{t+s}=T_{t} \cdot T_{s}$ for $t, s \geq 0$ and $T_{0}=I$.

(ii) The family $\left\{T_{t}\right\}$ is strongly continuous in $t$ at every $t \geq 0$ :

$$
\lim _{s \downarrow 0}\left\|T_{t+s} f-T_{t} f\right\|=0 \quad \text { for each } f \in C_{0}(K) .
$$

(iii) The family $\left\{T_{t}\right\}$ is non-negative and contractive on $C_{0}(K)$ :

$$
f \in C_{0}(K), 0 \leq f(x) \leq 1 \text { on } K \Longrightarrow 0 \leq T_{t} f(x) \leq 1 \text { on } K \text { for all } t \geq 0 .
$$

If $\left\{T_{t}\right\}_{t>0}$ is a Feller semigroup on the state space $K$, we define its infinitesimal generator $\mathfrak{A}$ by the formula

$$
\mathfrak{A} u=\lim _{t \downarrow 0} \frac{T_{t} u-u}{t},
$$

provided that the limit $(3.1)$ exists in $C_{0}(K)$.

The next theorem is the Hille-Yosida theorem [45] adapted to the present context (see [32, Theorem 9.3.1 and Corollary 9.3.2], [35, Theorem 9.35]): 
Theorem 3.1 (Hille-Yosida) Let $K$ be a locally compact, separable metric space. Then we have the following two assertions (i) and (ii).

(i) Let $\left\{T_{t}\right\}_{t>0}$ be a Feller semigroup on the state space $K$ and $\mathfrak{A}$ its infinitesimal generator. Then we have the following four assertions (a), (b), (c) and (d):

(a) The domain $D(\mathfrak{A})$ is dense in the Banach space $C_{0}(K)$.

(b) For each $\alpha>0$, the equation $(\alpha I-\mathfrak{A}) u=f$ has a unique solution $u$ in $D(\mathfrak{A})$ for any $f \in C_{0}(K)$. Hence, for each $\alpha>0$, the Green operator

$$
(\alpha I-\mathfrak{A})^{-1}: C_{0}(K) \longrightarrow C_{0}(K)
$$

can be defined by the formula

$$
u=(\alpha I-\mathfrak{A})^{-1} f \quad \text { for } f \in C_{0}(K) .
$$

(c) For each $\alpha>0$, the Green operator $(\alpha I-\mathfrak{A})^{-1}$ is non-negative on the space $C_{0}(K)$ :

$$
f \in C_{0}(K), f \geq 0 \quad \text { on } K \Longrightarrow(\alpha I-\mathfrak{A})^{-1} f \geq 0 \quad \text { on } K .
$$

(d) For each $\alpha>0$, the Green operator $(\alpha I-\mathfrak{A})^{-1}$ is bounded on the space $C_{0}(K)$ with norm

$$
\left\|(\alpha I-\mathfrak{A})^{-1}\right\| \leq \frac{1}{\alpha} .
$$

(ii) Conversely, if $\mathfrak{A}$ is a linear operator from $C_{0}(K)$ into itself satisfying condition (a) and if there is a constant $\alpha_{0} \geq 0$ such that, for all $\alpha>\alpha_{0}$, conditions (b) through (d) are satisfied, then $\mathfrak{A}$ is the infinitesimal generator of some Feller semigroup $\left\{T_{t}\right\}_{t \geq 0}$ on the state space $K$.

The next corollary plays an important role in the proof of Lemma 8.3 in Section 8 (see [35, Corollary 2.17]):

Corollary 3.1 Let $A$ be the infinitesimal generator of a Feller semigroup on a compact metric space $K$. Assume that the constant function 1 belongs to the domain $D(A)$ of $A$ and further that we have, for some constant $c$,

$$
(A 1)(x) \leq-c \text { on } K
$$

Then the operator $B=A+c I$ is the infinitesimal generator of some Feller semigroup on the state space $K$.

Following [32], we recall two useful criteria in terms of the positive maximum principle in order that a linear operator is the infinitesimal generator of some Feller semigroup (see [32, Theorem 9.3.3 and Corollary 9.3.4], [35, Theorem 9.50 and Corollary 9.51]):

Theorem 3.2 (Hille-Yosida-Ray) Let $K$ be a compact metric space. Then we have the following two assertions (i) and (ii).

(i) Let $B$ be a linear operator from the Banach space $C(K)=C_{0}(K)$ into itself, and assume that the following two conditions $(\alpha)$ and $(\beta)$ are satisfied:

$(\alpha)$ The domain $D(B)$ of $B$ is dense in $C(K)$. 
( $\beta$ ) There exists an open and dense subset $K_{0}$ of $K$ such that if $u \in D(B)$ takes a positive maximum at a point $x_{0}$ of $K_{0}$, then we have the inequality

$$
B u\left(x_{0}\right) \leq 0 .
$$

Then the operator $B$ is closable in $C(K)$.

(ii) Let $B$ be as in part (i), and further assume that the following two conditions $\left(\beta^{\prime}\right)$ and $(\gamma)$ are satisfied:

$\left(\beta^{\prime}\right)$ If $u \in D(B)$ takes a positive maximum at a point $x^{\prime}$ of $K$, then we have the inequality

$$
B u\left(x^{\prime}\right) \leq 0 .
$$

$(\gamma)$ For some $\alpha_{0} \geq 0$, the range $R\left(\alpha_{0} I-B\right)$ of $\alpha_{0} I-B$ is dense in $C(K)$.

Then the minimal closed extension $\bar{B}$ of $B$ is the infinitesimal generator of some Feller semigroup on the state space $K$.

The next corollary plays an important role in the proof of Lemma 8.2 in Section 8 (see [35, Corollary 2.19]):

Corollary 3.2 Let $A$ be the infinitesimal generator of a Feller semigroup $\left\{T_{t}\right\}_{t \geq 0}$ on a compact metric space $K$ and let $M$ be a bounded linear operator on the Banach space $C(K)$ into itself. If either $M$ or $C=A+M$ satisfies condition $\left(\beta^{\prime}\right)$ of Theorem 3.2, then the operator $C$ is the infinitesimal generator of some Feller semigroup on the state space $K$.

\section{The Dirichlet Problem}

An open set $\Omega$ in $\mathbf{R}^{N}$ is said to be of class $C^{1,1}$ if its boundary $\partial \Omega$ can be locally represented as the graph of a $C^{1}$ function whose first-order partial derivatives are all Lipschitz continuous. It should be emphasized that VMO functions are invariant under $C^{1,1}$-diffeomorphisms (see [1, Proposition 1.3]).

Let $\Omega$ be a bounded domain in Euclidean space $\mathbf{R}^{N}, N \geq 3$, with boundary $\partial \Omega$ of class $C^{1,1}$. If $1<p<\infty$, we define the usual Sobolev space $W^{2, p}(\Omega)$, and the boundary space $B^{2-1 / p, p}(\partial \Omega)$ of the boundary values $\gamma_{0} u$ of functions $u \in W^{2, p}(\Omega)$. It is known (see [2], [5], [40]) that the space $B^{2-1 / p, p}(\partial \Omega$ ) is a Besov space.

In this section we consider the following non-homogeneous Dirichlet problem: Given functions $f(x)$ and $\varphi\left(x^{\prime}\right)$ defined in $\Omega$ and on $\partial \Omega$, respectively, find a function $u(x)$ in $\Omega$ such that

$$
\begin{cases}A u=f & \text { in } \Omega, \\ \gamma_{0} u=\varphi & \text { on } \partial \Omega .\end{cases}
$$

The next existence and uniqueness theorem is a generalization of Bony [7, Théorème 3] to the VMO case (see [33, Theorem 4.1]):

Theorem 4.1 Let $N<p<\infty$, and assume that $c(x) \leq 0$ for almost all $x \in \Omega$. Then the non-homogeneous Dirichlet problem (4.1) has a unique solution $u \in W^{2, p}(\Omega)$ for any $f \in L^{p}(\Omega)$ and any $\varphi \in B^{2-1 / p, p}(\partial \Omega)$. 
If we associate with problem (4.1) a continuous linear operator

$$
\mathcal{A}_{D}=\left(A, \gamma_{0}\right): W^{2, p}(\Omega) \longrightarrow L^{p}(\Omega) \oplus B^{2-1 / p, p}(\partial \Omega)
$$

then we obtain from Theorem 4.1 that the mapping $\mathcal{A}_{D}$ is an algebraic and topological isomorphism. Indeed, the continuity of the inverse of $\mathcal{A}_{D}$ follows from an application of Banach's closed graph theorem (see [30, Theorem 3.10], [45, Chapter II, Section 6, Theorem 1]).

\section{The Oblique Derivative Problem}

The purpose of this section is to prove an existence and uniqueness theorem for the non-homogeneous oblique derivative problem in the framework of Sobolev spaces of $L^{p}$ type under condition (H.1) (Theorem 5.1).

5.1 Formulation of the Oblique Derivative Problem

In this subsection, we consider an oblique derivative boundary operator of the form

$$
L_{\nu} u:=\mu\left(x^{\prime}\right) \frac{\partial u}{\partial \mathbf{n}}+\beta\left(x^{\prime}\right) \cdot \partial_{x^{\prime}} u+\gamma\left(x^{\prime}\right) u
$$

Our starting point is the following existence and uniqueness theorem for the non-homogeneous oblique derivative problem with VMO coefficients under condition (H.1):

Theorem 5.1 Let $N<p<\infty$ and $\alpha>0$. Assume that condition (H.1) is satisfied. Then, for any $f \in L^{p}(\Omega)$ and any $\varphi \in B^{1-1 / p, p}(\partial \Omega)$ the non-homogeneous oblique derivative problem

$$
\begin{cases}(A-\alpha) u=f & \text { in } \Omega, \\ L_{\nu} u=\varphi & \text { on } \partial \Omega\end{cases}
$$

has a unique solution $u \in W^{2, p}(\Omega)$.

If we associate with problem (5.1) a linear operator

$$
\mathcal{A}_{\nu}(\alpha)=\left(A-\alpha, L_{\nu}\right): W^{2, p}(\Omega) \longrightarrow L^{p}(\Omega) \oplus B^{1-1 / p, p}(\partial \Omega),
$$

then we obtain from the trace theorem (see [2, Remarks 7.45], [40, p. 200, Theorem]) and Theorem 5.1 that the mapping $\mathcal{A}_{\nu}(\alpha)$ is an algebraic and topological isomorphism for any $\alpha>0$. Indeed, the continuity of the inverse of $\mathcal{A}_{\nu}(\alpha)$ follows from an application of Banach's closed graph theorem (see [30, Theorem 3.10], [45, Chapter II, Section 6, Theorem 1]). 


\subsection{Proof of Theorem 5.1}

The proof of Theorem 5.1 is divided into two steps. First, since $\mathbf{n}$ is the unit inward normal to the boundary $\partial \Omega$, it follows (see Figure 1.2) that

$$
\left\langle\mu\left(x^{\prime}\right) \mathbf{n}+\beta\left(x^{\prime}\right), \mathbf{n}\right\rangle=\mu\left(x^{\prime}\right)\langle\mathbf{n}, \mathbf{n}\rangle=\mu\left(x^{\prime}\right) \quad \text { for all } x^{\prime} \in \partial \Omega .
$$

Therefore, in terms of the local coordinates (1.3) we find that condition (H.1) is equivalent to the condition that the directional derivative

$$
\mu\left(x^{\prime}\right) \frac{\partial}{\partial \mathbf{n}}+\beta\left(x^{\prime}\right) \cdot \partial_{x^{\prime}}=\mu\left(x^{\prime}\right) \frac{\partial}{\partial x_{N}}+\sum_{j=1}^{N-1} \beta^{j}\left(x^{\prime}\right) \frac{\partial}{\partial x_{j}}
$$

associated with $L_{\nu}$ is nowhere tangential to the boundary $\partial \Omega$.

\subsubsection{Oblique Derivative Problems and the Distance Function}

First, we introduce the distance function $d(x)$ by the formula

$$
d(x)=\operatorname{dist}(x, \partial \Omega) \quad \text { for } x \in \bar{\Omega} .
$$

It is easy to see that $d(x)$ is uniformly Lipschitz continuous. In fact, we have, by the triangle inequality,

$$
|d(x)-d(y)| \leq|x-y| \quad \text { for all } x, y \in \bar{\Omega} .
$$

Furthermore, for $a>0$ we define a tubular neighborhood $\Gamma_{a}$ of $\partial \Omega$ by the formula

$$
\Gamma_{a}:=\{x \in \bar{\Omega}: d(x) \leq a\} .
$$

Then we find from the proof of [19, Lemma 14.16] that

$$
d(x) \in C^{1,1}\left(\Gamma_{a_{0}}\right) \quad \text { for some } a_{0}>0 .
$$

It should be noticed that the points $x \in \Gamma_{a_{0}}$ and $y^{\prime} \in \partial \Omega$ are related as follows:

$$
x=y^{\prime}+d(x) \mathbf{n}\left(y^{\prime}\right),
$$

where $\mathbf{n}\left(y^{\prime}\right)$ is the unit inward normal to $\partial \Omega$ at $y^{\prime}$.

By using [23, Lemma 1.67], we can extend the distance function $d(x)$ in such a way that

$$
d(x) \in C^{1,1}(\bar{\Omega}) .
$$

Hence, it follows from an application of Sobolev imbedding theorem ([2, Theorem 4.12, Part II $]$ ) and Rademacher's theorem ([23, Corollary 1.73]) that

$$
d(x) \in W^{2, \infty}(\Omega)=C^{1,1}(\bar{\Omega}) .
$$

Finally, we have the formula

$$
\operatorname{grad} d\left(x^{\prime}\right)=\mathbf{n}\left(x^{\prime}\right) \quad \text { at every point } x^{\prime} \in \partial \Omega .
$$

Now we are in a position to study the non-homogeneous oblique derivative problem via the distance function (cf. [24, Theorem 4.1], [25, Theorem 2.3.5]): 
Theorem 5.2 Let $N<p<\infty$ and

$$
A_{0} u:=\sum_{i, j=1}^{N} a^{i j}(x) \frac{\partial^{2} u}{\partial x_{i} \partial x_{j}} .
$$

If condition (H.1) is satisfied, then there exists a constant $\alpha_{0}>0$ (see formula (5.8) below) such that the non-homogeneous oblique derivative problem

$$
\begin{cases}\left(A_{0}-\alpha_{0}\right) u=f & \text { in } \Omega, \\ L_{\nu} u=\varphi & \text { on } \partial \Omega\end{cases}
$$

has a unique solution $u \in W^{2, p}(\Omega)$ for any $f \in L^{p}(\Omega)$ and any $\varphi \in B^{1-1 / p, p}(\partial \Omega)$.

Proof The proof of Theorem 5.2 is divided into three steps.

Step (1): If we let

$$
u(x)=v(x) e^{-d(x)} \quad \text { for } x \in \bar{\Omega},
$$

then we have, by a direct calculation (cf. [25, pp. 138-139]),

$$
\left(A_{0}-\alpha\right) u=f \quad \text { in } \Omega \Longleftrightarrow\left(\widetilde{A}_{0}-\alpha\right) v=f e^{d(x)} \quad \text { in } \Omega .
$$

Here the differential operator $\widetilde{A}_{0}$ is given by the formula

$$
\begin{aligned}
\widetilde{A}_{0} v= & \sum_{i, j=1}^{N} a^{i j}(x) \frac{\partial^{2} v}{\partial x_{i} \partial x_{j}}+\sum_{i=1}^{N} \widetilde{b}^{i}(x) \frac{\partial v}{\partial x_{i}}+\widetilde{c}(x) v(x) \\
:= & \sum_{i, j=1}^{N} a^{i j}(x) \frac{\partial^{2} v}{\partial x_{i} \partial x_{j}}-2 \sum_{i=1}^{N}\left(\sum_{j=1}^{N} a^{i j}(x) \frac{\partial d}{\partial x_{j}}\right) \frac{\partial v}{\partial x_{i}} \\
& +\left(\sum_{i, j=1}^{N} a^{i j}(x) \frac{\partial d}{\partial x_{i}} \frac{\partial d}{\partial x_{j}}-\sum_{i, j=1}^{N} a^{i j}(x) \frac{\partial^{2} d}{\partial x_{i} \partial x_{j}}\right) .
\end{aligned}
$$

By assertion (5.2), we remark that the coefficients $\widetilde{b}^{i}(x)$ and $\widetilde{c}(x)$ satisfy the following regularity conditions:

$$
\begin{aligned}
& \text { - } \widetilde{b}^{i}(x)=-2 \sum_{j=1}^{N} a^{i j}(x) \frac{\partial d}{\partial x_{j}} \in L^{\infty}(\Omega) \quad \text { for } 1 \leq i \leq N, \\
& \text { - } \widetilde{c}(x)=\sum_{i, j=1}^{N} a^{i j}(x) \frac{\partial d}{\partial x_{i}} \frac{\partial d}{\partial x_{j}}-\sum_{i, j=1}^{N} a^{i j}(x) \frac{\partial^{2} d}{\partial x_{i} \partial x_{j}} \in L^{\infty}(\Omega) .
\end{aligned}
$$

Therefore, if we let

$$
\alpha_{0}:=\max _{x \in \bar{\Omega}}\left|\sum_{i, j=1}^{N} a^{i j}(x)\left(\frac{\partial d}{\partial x_{i}} \frac{\partial d}{\partial x_{j}}-\frac{\partial^{2} d}{\partial x_{i} \partial x_{j}}\right)\right|,
$$

then we have the inequality

$$
\widetilde{c}(x)-\alpha_{0} \leq 0 \quad \text { on } \bar{\Omega} .
$$


Step (2): On the other hand, by using assertions (5.2) and (5.3) we have the formula

$$
\begin{aligned}
& L_{\nu} u=\mu\left(x^{\prime}\right) \frac{\partial u}{\partial \mathbf{n}}+\beta\left(x^{\prime}\right) \cdot \partial_{x^{\prime}} u+\gamma\left(x^{\prime}\right) u=\varphi \quad \text { on } \partial \Omega \\
& \Longleftrightarrow \\
& \widetilde{L}_{\nu} v:=\mu\left(x^{\prime}\right) \frac{\partial v}{\partial \mathbf{n}}+\beta\left(x^{\prime}\right) \cdot \partial_{x^{\prime}} v+\widetilde{\gamma}\left(x^{\prime}\right) v=\varphi \quad \text { on } \partial \Omega,
\end{aligned}
$$

where

$$
\widetilde{\gamma}\left(x^{\prime}\right)=\gamma\left(x^{\prime}\right)-\mu\left(x^{\prime}\right) \quad \text { on } \partial \Omega .
$$

Indeed, it suffices to note the following:

$$
\begin{cases}\frac{\partial}{\partial \mathbf{n}}\left(e^{d(x)}\right)=\left\langle\mathbf{n}, \operatorname{grad} d\left(x^{\prime}\right)\right\rangle e^{d\left(x^{\prime}\right)}=\langle\mathbf{n}, \mathbf{n}\rangle=1 & \text { on } \partial \Omega \\ \beta\left(x^{\prime}\right) \cdot \partial_{x^{\prime}}\left(e^{d(x)}\right)=0 & \text { on } \partial \Omega\end{cases}
$$

Step (3): By combining formulas (5.5) and (5.10), we have the equivalence of the two oblique derivative problems (5.4) and (5.12):

$$
\begin{gathered}
\begin{cases}\left(A_{0}-\alpha_{0}\right) u=f & \text { in } \Omega, \\
L_{\nu} u=\varphi & \text { on } \partial \Omega\end{cases} \\
\Longleftrightarrow \\
\begin{cases}\left(\widetilde{A}_{0}-\alpha_{0}\right) v=f e^{d(x)} & \text { in } \Omega, \\
\widetilde{L}_{\nu} v=\varphi & \text { on } \partial \Omega .\end{cases}
\end{gathered}
$$

By assertions (5.6) and (5.7) and inequality (5.9), it should be noticed that the coefficients $\widetilde{b}^{i}(x)$ and $\widetilde{c}(x)$ of the differential operator $\widetilde{A}_{0}$ satisfies the following two conditions:

(2) $\widetilde{b}^{i}(x) \in L^{\infty}(\Omega)$ for $1 \leq i \leq N$.

(3) $\widetilde{c}(x) \in L^{\infty}(\Omega)$ and $\widetilde{c}(x)-\alpha_{0} \leq 0$ for almost all $x \in \Omega$.

By formula (5.11), it follows that the coefficients $\mu\left(x^{\prime}\right)$ and $\widetilde{\gamma}\left(x^{\prime}\right)$ of the boundary condition $\widetilde{L}_{\nu}$ satisfies the following two conditions (H.1) and $\widetilde{(H .2)}$ :

(H.1) $\mu\left(x^{\prime}\right)>0$ on $\partial \Omega$.

(H.2) $\widetilde{\gamma}\left(x^{\prime}\right)=\gamma\left(x^{\prime}\right)-\mu\left(x^{\prime}\right) \leq-\mu\left(x^{\prime}\right)<0$ on $\partial \Omega$.

Therefore, by applying [34, Theorem 4.1] (cf. [24, Theorem 4.1], [25, Theorem 2.3.5]) with

$$
\begin{aligned}
& A:=\widetilde{A}_{0}, \quad c(x):=\widetilde{c}(x)-\alpha_{0}, \quad \alpha:=0, \\
& f:=f e^{d(x)}, \\
& L_{0}:=\widetilde{L}_{\nu}, \quad \gamma\left(x^{\prime}\right):=\widetilde{\gamma}\left(x^{\prime}\right),
\end{aligned}
$$

we obtain that the non-homogeneous oblique derivative problem (5.12) has a unique solution $v \in W^{2, p}(\Omega)$ for any $f e^{d(x)} \in L^{p}(\Omega)$ and any $\varphi \in B^{1-1 / p, p}(\partial \Omega)$.

Rephrased, the non-homogeneous oblique derivative problem (5.4) has a unique solution $u=v e^{-d(x)} \in W^{2, p}(\Omega)$ for any $f \in L^{p}(\Omega)$ and any $\varphi \in B^{1-1 / p, p}(\partial \Omega)$. Indeed, we have, by assertion (5.2),

$$
u=v e^{-d(x)} \in W^{2, p}(\Omega) \quad \text { for } v \in W^{2, p}(\Omega) .
$$

Now the proof of Theorem 5.2 is complete. 


\subsubsection{End of Proof of Theorem 5.1}

The proof of Theorem 5.1 is divided into five steps.

Step (1): If we associate with problem (5.4) a continuous linear operator

$$
\mathcal{A}_{0}\left(\alpha_{0}\right)=\left(A_{0}-\alpha_{0}, L_{\nu}\right): W^{2, p}(\Omega) \longrightarrow L^{p}(\Omega) \oplus B^{1-1 / p, p}(\partial \Omega),
$$

then we obtain from Theorem 5.2 that the mapping $\mathcal{A}_{0}\left(\alpha_{0}\right)$ is an algebraic and topological isomorphism. In particular, we have the assertion

$$
\text { ind } \mathcal{A}_{0}\left(\alpha_{0}\right)=\operatorname{dim} N\left(\mathcal{A}_{0}\left(\alpha_{0}\right)\right)-\operatorname{codim} R\left(\mathcal{A}_{0}\left(\alpha_{0}\right)\right)=0 \text {. }
$$

Step (2): If we let

$$
B\left(\alpha_{0}-\alpha\right) u:=\sum_{i=1}^{N} b^{i}(x) \frac{\partial u}{\partial x_{i}}+\left(c(x)+\alpha_{0}-\alpha\right) u \quad \text { for } \alpha>0,
$$

then it follows that the operator

$$
B\left(\alpha_{0}-\alpha\right): W^{2, p}(\Omega) \longrightarrow W^{1, p}(\Omega)
$$

is continuous for $1<p<\infty$. However, it follows from an application of the RellichKondrachov theorem (see [2, Theorem 6.3, Part II], [19, Theorem 7.26]) that the injection

$$
W^{1, p}(\Omega) \longrightarrow L^{p}(\Omega)
$$

is compact for $1<p<\infty$. Hence we find that the mapping

$$
B\left(\alpha_{0}-\alpha\right): W^{2, p}(\Omega) \longrightarrow L^{p}(\Omega)
$$

is compact for $1<p<\infty$. It should be noticed that

$$
\mathcal{A}_{\nu}(\alpha)=\left(A-\alpha, L_{\nu}\right)=\left(A_{0}-\alpha_{0}, L_{\nu}\right)+\left(B\left(\alpha_{0}-\alpha\right), 0\right)=\mathcal{A}_{0}\left(\alpha_{0}\right)+\left(B\left(\alpha_{0}-\alpha\right), 0\right) .
$$

However, we know (see [20, Theorem 2.6], [30, Theorem 5.10]) that the index is stable under compact perturbations.

Therefore, we obtain that the mapping

$$
\mathcal{A}_{\nu}(\alpha)=\mathcal{A}_{0}\left(\alpha_{0}\right)+\left(B\left(\alpha_{0}-\alpha\right), 0\right): W^{2, p}(\Omega) \longrightarrow L^{p}(\Omega) \oplus B^{1-1 / p, p}(\partial \Omega)
$$

is a Fredholm operator with index zero, for any $\alpha>0$, since we have, by assertion (5.13),

$$
\text { ind } \mathcal{A}_{\nu}(\alpha)=\text { ind } \mathcal{A}_{0}\left(\alpha_{0}\right)=0 \text {. }
$$

In particular, the Fredholm alternative holds true for the operator $\mathcal{A}_{\nu}(\alpha)$ for any $\alpha>0$.

Step (3): On the other hand, the uniqueness result in Theorem 5.1 follows from an application of the Bakel'man-Aleksandrov maximum principle (see [22, Corollary 2.4], [34, Theorem 4.3])): 
Theorem 5.3 (the maximum principle) Let $N<p<\infty$ and $\alpha>0$. Assume that condition (H.1) is satisfied. If a function $u \in W^{2, p}(\Omega)$ satisfies the conditions

$$
\begin{cases}(A-\alpha) u \leq 0 & \text { almost everywhere in } \Omega, \\ L_{\nu} u \leq 0 & \text { on } \partial \Omega\end{cases}
$$

then it follows that either $u(x)$ is a non-negative constant function or $u(x)>0$ on $\bar{\Omega}$.

Step (4): By applying Theorem 5.3 to the two functions $\pm u(x)$, it follows that we have, for $\alpha>0$,

$$
\left\{\begin{array}{ll}
(A-\alpha) u=0 & \text { almost everywhere in } \Omega \\
L_{\nu} u=0 & \text { on } \partial \Omega
\end{array} \quad \Longrightarrow u=0 \quad \text { in } \Omega .\right.
$$

This proves that the mapping

$$
\mathcal{A}_{\nu}(\alpha)=\left(A-\alpha, L_{\nu}\right): W^{2, p}(\Omega) \longrightarrow L^{p}(\Omega) \oplus B^{1-1 / p, p}(\partial \Omega)
$$

is injective for any $\alpha>0$ if $N<p<\infty$. Hence it is also surjective for any $\alpha>0$ if $N<p<\infty$, since we have, by assertion (5.14),

$$
\text { ind } \mathcal{A}_{\nu}(\alpha)=\operatorname{dim} N\left(\mathcal{A}_{\nu}(\alpha)\right)-\operatorname{codim} R\left(\mathcal{A}_{\nu}(\alpha)\right)=0 .
$$

Step (5): Summing up, we have proved that the mapping

$$
\mathcal{A}_{\nu}(\alpha)=\left(A-\alpha, L_{\nu}\right): W^{2, p}(\Omega) \longrightarrow L^{p}(\Omega) \oplus B^{1-1 / p, p}(\partial \Omega)
$$

is an algebraic and topological isomorphism for any $\alpha>0$ if $N<p<\infty$, since the continuity of the inverse of $\mathcal{A}_{\nu}(\alpha)$ follows from an application of Banach's closed graph theorem (see [30, Theorem 3.10], [45, Chapter II, Section 6, Theorem 1]).

Now the proof of Theorem 5.1 is complete.

\section{Proof of Theorem 1.2}

This section is devoted to the proof of Theorem 1.2 under condition (H.1). In order to prove Theorem 1.2, it suffices to verify all conditions (a) through (d) in the Hille-Yosida theorem (Theorem 3.1) with

$$
K:=\bar{\Omega}, \quad C_{0}(K):=C(\bar{\Omega}), \quad \mathfrak{A}:=\mathfrak{A}_{\nu}
$$

The proof of Theorem 1.2 is divided into four steps.

Step (1): First, we prove that, for each $\alpha>0$, the equation $(\alpha I-A) u=f$ has a unique solution $u \in D\left(\mathfrak{A}_{N}\right)$ for any $f \in C(\bar{\Omega})$.

By applying Theorem 5.1, we obtain that the oblique derivative problem

$$
\begin{cases}(\alpha-A) u=f & \text { almost everywhere in } \Omega \\ L_{\nu} u=0 & \text { on } \partial \Omega\end{cases}
$$

has a unique solution $u \in W^{2, p}(\Omega)$ for any $f \in L^{p}(\Omega)$ with $N<p<\infty$. In particular, for any $f \in C(\bar{\Omega})$ there exists a function $u \in W^{2, p}(\Omega)$ such that

$$
(\alpha-A) u=f \quad \text { in } \Omega .
$$


Hence we have the assertion

$$
A u=\alpha u-f \in C(\bar{\Omega}) .
$$

By formula (1.6), this proves that

$$
\left\{\begin{array}{l}
u \in D\left(\mathfrak{A}_{\nu}\right), \\
\left(\alpha I-\mathfrak{A}_{\nu}\right) u=f .
\end{array}\right.
$$

Step (2): Secondly, we prove that, for each $\alpha>0$, the Green operator $G_{\alpha}^{\nu}=$ $\left(\alpha I-\mathfrak{A}_{\nu}\right)^{-1}$ is non-negative on the space $C(\bar{\Omega})$ :

$$
f \in C(\bar{\Omega}), f(x) \geq 0 \quad \text { in } \Omega \Longrightarrow u(x)=G_{\alpha}^{\nu} f(x) \geq 0 \quad \text { in } \Omega .
$$

More precisely, we prove the following assertion:

$$
f \in C(\bar{\Omega}), f(x) \geq 0, f(x) \not \equiv 0 \quad \text { in } \Omega \Longrightarrow u(x)=G_{\alpha}^{\nu} f(x)>0 \quad \text { on } \bar{\Omega} .
$$

The situation can be visualized as follows:

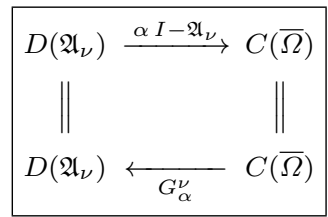

Fig. 6.1 The operators $\alpha I-\mathfrak{A}_{\nu}$ and $G_{\alpha}^{\nu}=\left(\alpha I-\mathfrak{A}_{\nu}\right)^{-1}$ for $\alpha>0$

Since we have the formulas

$$
\begin{cases}u \in W^{2, p}(\Omega) & \text { for } N<p<\infty, \\ (A-\alpha) u=-f \leq 0 & \text { almost everywhere in } \Omega, \\ L_{\nu} u=0 & \text { on } \partial \Omega,\end{cases}
$$

by applying Theorem 5.3 (the maximum principle) we obtain that either $u(x)$ is a non-negative constant function or $u(x)>0$ on $\bar{\Omega}$. However, if $u(x) \equiv 0$ in $\Omega$, then it follows that

$$
f(x)=(\alpha-A) u(x) \equiv 0 \quad \text { in } \Omega .
$$

This contradiction proves that either $u(x)$ is a positive constant function or $u(x)>$ 0 on $\bar{\Omega}$, that is,

$$
G_{\alpha}^{\nu} f(x)>0 \text { on } \bar{\Omega} \text {. }
$$

Step (3): Thirdly, we prove that, for each $\alpha>0$, the Green operator $G_{\alpha}^{\nu}=$ $\left(\alpha I-\mathfrak{A}_{\nu}\right)^{-1}$ is bounded on the space $C(\bar{\Omega})$ with norm $1 / \alpha$ :

$$
\left\|G_{\alpha}^{\nu}\right\| \leq \frac{1}{\alpha} \quad \text { for all } \alpha>0 .
$$

By virtue of assertion (6.1), it suffices to show that

$$
\alpha G_{\alpha}^{\nu} 1(x) \leq 1 \quad \text { on } \bar{\Omega} .
$$


If we let

$$
v(x):=\alpha G_{\alpha}^{\nu} 1(x)-1,
$$

then we have the assertions

$$
\begin{cases}u \in W^{2, p}(\Omega) & \text { for } N<p<\infty, \\ (A-\alpha) v=0 & \text { in } \Omega \\ L_{\nu} v=0 & \text { on } \partial \Omega\end{cases}
$$

By applying Theorem 5.3 to the function $u(x):=-v(x)$, we arrive at a contradiction that

$$
\max _{\bar{\Omega}} v>0 \Longrightarrow v(x) \equiv 0 \quad \text { in } \Omega
$$

This proves that

$$
\max _{\bar{\Omega}} v \leq 0
$$

or equivalently,

$$
\alpha G_{\alpha}^{\nu} 1(x) \leq 1 \quad \text { on } \bar{\Omega} .
$$

Step (4): The closedness of $\mathfrak{A}_{\nu}$ is an immediate consequence of that of the inverse $G_{\alpha}^{\nu-1}=\alpha I-\mathfrak{A}_{\nu}$ (see [45, p. 79, Proposition 3]).

Step (5): Finally, we prove that the domain $D\left(\mathfrak{A}_{\nu}\right)$ is dense in $C(\bar{\Omega})$. More precisely, we prove that, for each $u \in C(\bar{\Omega})$, we have the assertion

$$
\lim _{\alpha \rightarrow+\infty}\left\|\alpha G_{\alpha}^{\nu} u-u\right\|_{C(\bar{\Omega})}=0 .
$$

It suffices to prove assertion (6.3) for any $v \in C^{1,1}(\bar{\Omega})$ such that $L_{\nu} v=0$ on $\partial \Omega$. In fact, we have the following (see [4, Lemma 3.2], [34, Lemma 4.4])):

Lemma 6.1 Let $u \in C(\bar{\Omega})$. For any given $\varepsilon>0$, we can find a function $v \in C^{1,1}(\bar{\Omega})=$ $W^{2, \infty}(\Omega)$ such that

$$
\left\{\begin{array}{l}
\|u-v\|_{C(\bar{\Omega})}<\varepsilon \\
L_{\nu} v=0 \text { on } \partial \Omega
\end{array}\right.
$$

In order to prove assertion (6.3) for any $v \in C^{1,1}(\bar{\Omega})$ such that $L_{\nu} v=0$, we introduce an extension $\widetilde{G_{\alpha}^{\nu}}$ of the Green operator $G_{\alpha}^{\nu}$ to the space $L^{\infty}(\Omega)$ for $N<p<\infty$. By Theorem 5.1, we find that the oblique derivative problem

$$
\begin{cases}(\alpha-A) u=f & \text { almost everywhere in } \Omega, \\ L_{\nu} u=0 & \text { on } \partial \Omega\end{cases}
$$

has a unique solution $u \in W^{2, p}(\Omega)$ for any $f \in L^{p}(\Omega)$. If we let

$$
u:=\widetilde{G_{\alpha}^{\nu}} f \quad \text { for } f \in L^{\infty}(\Omega),
$$

then it is easy to verify that the operator $\widetilde{G_{\alpha}^{\nu}}$ is an extension of $G_{\alpha}^{\nu}$ to $L^{\infty}(\Omega)$. Moreover, just as in the proof of assertions (6.1) and (6.2) we can prove the following two assertions (A) and (B):

(A) The operator $\widetilde{G_{\alpha}^{\nu}}: L^{\infty}(\Omega) \rightarrow C(\bar{\Omega})$ is non-negative. 


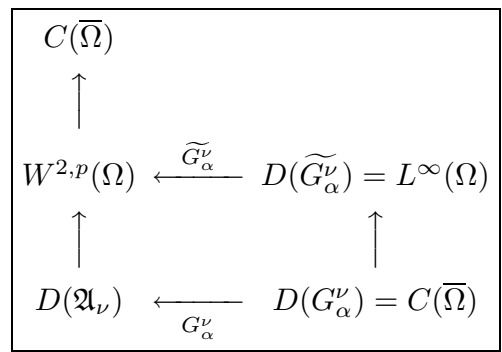

Fig. 6.2 The mapping properties of the Green operators $G_{\alpha}^{\nu}$ and $\widetilde{G_{\alpha}^{\nu}}$ for $\alpha>0$

(B) The operator $\widetilde{G_{\alpha}^{\nu}}: L^{\infty}(\Omega) \rightarrow C(\bar{\Omega})$ is bounded with norm $1 / \alpha$ :

$$
\left\|\widetilde{G_{\alpha}^{\nu}}\right\| \leq \frac{1}{\alpha} \quad \text { for all } \alpha>0
$$

First, since $a^{i j}(x), b^{i}(x), c(x) \in L^{\infty}(\Omega)$ and $v \in C^{1,1}(\bar{\Omega})=W^{2, \infty}(\Omega)$, it follows that

$$
A v=\sum_{i, j=1}^{N} a^{i j}(x) \frac{\partial^{2} v}{\partial x_{i} \partial x_{j}}+\sum_{i=1}^{N} b^{i}(x) \frac{\partial v}{\partial x_{i}}+c(x) v \in L^{\infty}(\Omega) .
$$

Thus, if we let

$$
w:=\alpha G_{\alpha}^{\nu} v-\widetilde{G_{\alpha}^{\nu}}(A v),
$$

then we have the assertions (see Figure 6.2)

$$
\begin{cases}w \in W^{2, p}(\Omega) & \text { for } N<p<\infty, \\ (A-\alpha) w=(A-\alpha) v & \text { almost everywhere in } \Omega, \\ L_{\nu} w=0 & \text { on } \partial \Omega,\end{cases}
$$

and so

$$
\begin{cases}w-v \in W^{2, p}(\Omega) & \text { for } N<p<\infty, \\ (A-\alpha)(w-v)=0 & \text { almost everywhere in } \Omega, \\ L_{\nu}(w-v)=0 & \text { on } \partial \Omega .\end{cases}
$$

By applying Theorem 5.1 to the function $w(x)-v(x)$, we obtain that $w-v=0$ in $\Omega$. This implies that

$$
v=w=\alpha G_{\alpha}^{\nu} v-\widetilde{G_{\alpha}^{\nu}}(A v) .
$$

Therefore, the desired assertion (6.3) for any $v \in C^{1,1}(\bar{\Omega})$ such that $L_{\nu} v=0$ follows from an application of assertions (6.5) and (6.6), since we have, for all $\alpha>0$,

$$
\left\|v-\alpha G_{\alpha}^{\nu} v\right\|_{C(\bar{\Omega})}=\left\|\widetilde{G_{\alpha}^{\nu}}(A v)\right\|_{C(\bar{\Omega})} \leq \frac{1}{\alpha}\|A v\|_{L^{\infty}(\Omega)} .
$$

Now the proof of Theorem 1.2 is complete. 


\section{Feller Semigroups and Boundary Value Problems}

The purpose of this section is to prove a general existence theorem for Feller semigroups in terms of boundary value problems (Theorem 7.2), following the main idea of Taira [32, Section 9.6] (see Bony-Courrège-Priouret [8] and SatoUeno [29]).

7.1 Green Operators and Harmonic Operators for the Dirichlet problem

Let $N<p<\infty$ and $\alpha>0$. Since we have the inequality

$$
c(x)-\alpha \leq-\alpha \text { for almost all } x \in \Omega,
$$

by applying Theorem 4.1 to the operator $A-\alpha$ we obtain that, for any $f \in C(\bar{\Omega})$ and any $\varphi \in C^{2}(\partial \Omega)$ the Dirichlet problem

$$
\begin{cases}(\alpha-A) u=f & \text { almost everywhere in } \Omega, \\ u=\varphi & \text { on } \partial \Omega\end{cases}
$$

has a unique solution $u \in W^{2, p}(\Omega)$. Indeed, it suffices to note that $C(\bar{\Omega}) \subset L^{p}(\Omega)$ and $C^{2}(\partial \Omega) \subset B^{2-1 / p, p}(\partial \Omega)$. Therefore, we can introduce two linear operators

$$
G_{\alpha}^{0}: C(\bar{\Omega}) \longrightarrow C(\bar{\Omega}),
$$

and

$$
H_{\alpha}: C^{2}(\partial \Omega) \longrightarrow C(\bar{\Omega})
$$

as follows.

(I) For any $f \in C(\bar{\Omega})$, the function $G_{\alpha}^{0} f \in W^{2, p}(\Omega) \cap W_{0}^{1, p}(\Omega)$ is the unique solution of the problem

$$
\begin{cases}(\alpha-A) G_{\alpha}^{0} f=f & \text { in } \Omega, \\ G_{\alpha}^{0} f=0 & \text { on } \partial \Omega .\end{cases}
$$

(II) For any $\varphi \in C^{2}(\partial \Omega)$, the function $H_{\alpha} \varphi \in W^{2, p}(\Omega)$ is the unique solution of the problem

$$
\begin{cases}(\alpha-A) H_{\alpha} \varphi=0 & \text { in } \Omega, \\ H_{\alpha} \varphi=\varphi & \text { on } \partial \Omega .\end{cases}
$$

Here it should be noticed that we have, by Sobolev's imbedding theorem (see [2, Theorem 4.12, Part II]),

$$
W^{2, p}(\Omega) \subset C^{2-N / p}(\bar{\Omega}) \subset C^{1}(\bar{\Omega}) \text { for } N<p<\infty,
$$

and also, by an imbedding theorem for Besov spaces (see [2, Theorem 7.34, Part (c)]),

$$
\left\{\begin{array}{l}
C^{1}(\partial \Omega) \subset B^{1-1 / p, p}(\partial \Omega) \subset C(\partial \Omega), \\
C^{2}(\partial \Omega) \subset B^{2-1 / p, p}(\partial \Omega) \subset C^{1}(\partial \Omega),
\end{array}\right.
$$

since $(1-1 / p) p=p-1>N-1$.

The operator $G_{\alpha}^{0}$ is called the Green operator and the operator $H_{\alpha}$ is called the harmonic operator, respectively.

Then we can prove the following theorem for the Dirichlet problem (see [35, Theorem 10.9]): 
Theorem 7.1 Let $\alpha>0$. Then we have the following two assertions for the operators $G_{\alpha}^{0}$ and $H_{\alpha}$.

(i) The Green operator

$$
G_{\alpha}^{0}: C(\bar{\Omega}) \longrightarrow C(\bar{\Omega})
$$

enjoys the following properties (a) through $(d)$ :

(a) The operator $G_{\alpha}^{0}$ is non-negative and bounded with norm

$$
\left\|G_{\alpha}^{0}\right\|=\left\|G_{\alpha}^{0} 1\right\|_{C(\bar{\Omega})} \leq \frac{1}{\alpha} .
$$

(b) For any $f \in C(\bar{\Omega})$, we have the assertion

$$
G_{\alpha}^{0} f=0 \quad \text { on } \partial \Omega .
$$

(c) For all $\alpha, \beta>0$, the resolvent equation holds true:

$$
G_{\alpha}^{0} f-G_{\beta}^{0} f+(\alpha-\beta) G_{\alpha}^{0}\left(G_{\beta}^{0} f\right)=0 \quad \text { for each } f \in C(\bar{\Omega}) .
$$

(d) For any $f \in C(\bar{\Omega})$, we have the assertion

$$
\lim _{\alpha \rightarrow+\infty} \alpha G_{\alpha}^{0} f(x)=f(x) \quad \text { for each } x \in \Omega .
$$

Furthermore, if $\left.f\right|_{\partial \Omega}=0$, that is, if $f \in C_{0}(\bar{\Omega})$, then this convergence is uniform in $x \in \bar{\Omega}$. In other words, we have the assertion

$$
\lim _{\alpha \rightarrow+\infty} \alpha G_{\alpha}^{0} f=f \quad \text { in } C_{0}(\bar{\Omega}) .
$$

(ii) The harmonic operator $H_{\alpha}: C^{2}(\partial \Omega) \rightarrow C(\bar{\Omega})$ can be uniquely extended to a nonnegative, bounded linear operator

$$
H_{\alpha}: C(\partial \Omega) \longrightarrow C(\bar{\Omega})
$$

that enjoys the following properties (e) through $(g)$ :

(e) The operator $H_{\alpha}$ is bounded with norm $\left\|H_{\alpha}\right\|=1$.

(f) For any $\varphi \in C(\partial \Omega)$, we have the assertion

$$
H_{\alpha} \varphi=\varphi \quad \text { on } \partial \Omega \text {. }
$$

(g) For all $\alpha, \beta>0$, we have the equation

$$
H_{\alpha} \varphi-H_{\beta} \varphi+(\alpha-\beta) G_{\alpha}^{0}\left(H_{\beta} \varphi\right)=0 \quad \text { for each } \varphi \in C(\partial \Omega) .
$$

Remark 7.1 The resolvent equation (7.7) remains valid for each $f \in L^{\infty}(\Omega)$ (see Figure 7.1 below):

$$
\widetilde{G_{\alpha}^{0}} f-\widetilde{G_{\beta}^{0}} f+(\alpha-\beta) G_{\alpha}^{0}\left(\widetilde{G_{\beta}^{0}} f\right)=0 \text { for each } f \in L^{\infty}(\Omega) .
$$

Indeed, since the function

$$
u:=\widetilde{G_{\alpha}^{0}} f-\widetilde{G_{\beta}^{0}} f+(\alpha-\beta) G_{\alpha}^{0}\left(\widetilde{G_{\beta}^{0}} f\right) \in W^{2, p}(\Omega) \cap W_{0}^{1, p}(\Omega)
$$

is a unique solution of the Dirichlet problem

$$
\begin{cases}(\alpha-A) u=0 & \text { almost everywhere in } \Omega, \\ u=0 & \text { on } \partial \Omega,\end{cases}
$$

it follows from an application of Theorem 4.1 with $A:=A-\alpha$ that

$$
\widetilde{G_{\alpha}^{0}} f-\widetilde{G_{\beta}^{0}} f+(\alpha-\beta) G_{\alpha}^{0}\left(\widetilde{G_{\beta}^{0}} f\right)=0 .
$$




$$
\begin{gathered}
D\left(\widetilde{G_{\alpha}^{0}}\right)=L^{\infty}(\Omega) \stackrel{\widetilde{G_{\alpha}^{0}}}{\longrightarrow} W^{2, p}(\Omega) \cap W_{0}^{1, p}(\Omega) \\
\uparrow \\
D\left(G_{\alpha}^{0}\right)=C(\bar{\Omega}) \stackrel{G_{\alpha}^{0}}{\longrightarrow} W^{2, p}(\Omega) \cap W_{0}^{1, p}(\Omega)
\end{gathered}
$$

Fig. 7.1 The Green operators $G_{\alpha}^{0}$ and $\widetilde{G_{\alpha}^{0}}$ for $\alpha>0$ in Theorem 7.1 and Remark 7.1

\subsection{General Boundary Value Problems}

Let $L$ be a first-order, Wentzell boundary condition of the form

$$
L u:=\mu\left(x^{\prime}\right) \frac{\partial u}{\partial \mathbf{n}}+\beta\left(x^{\prime}\right) \cdot \partial_{x^{\prime}} u+\gamma\left(x^{\prime}\right) u-\delta\left(x^{\prime}\right)\left(\left.A u\right|_{\partial \Omega}\right) \quad \text { on } \partial \Omega .
$$

In this subsection we consider the following first-order Wentzell boundary value problem in the framework of the spaces of continuous functions:

$$
\begin{cases}(\alpha-A) u=f & \text { in } \Omega, \\ L u=0 & \text { on } \partial \Omega .\end{cases}
$$

To do this, we introduce three linear operators associated with problem (7.11).

(I) First, we introduce a linear operator

$$
\bar{A}: C(\bar{\Omega}) \longrightarrow C(\bar{\Omega})
$$

as follows:

(a) The domain $D(\bar{A})$ of $\bar{A}$ is the space

$$
D(\bar{A})=\left\{u \in W^{2, p}(\Omega): A u \in C(\bar{\Omega})\right\} \quad \text { for } N<p<\infty .
$$

(b) $\bar{A} u=A u$ for every $u \in D(\bar{A})$.

Here $A u$ is taken in the sense of distributions.

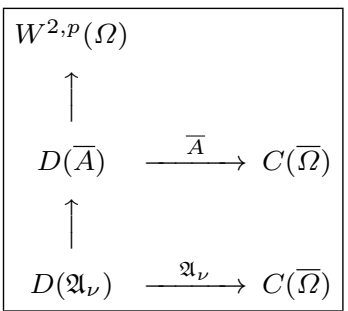

Fig. 7.2 The operators $\mathfrak{A}_{\nu}$ and $\bar{A}$ in the definitions (1.6) and (7.12)

Then we have the following lemma: 
Lemma 7.1 The operator $\bar{A}$ is a densely defined, closed linear operator in the space $C(\bar{\Omega})$.

Proof (1) First, by the definition (1.6) of $\mathfrak{A}_{\nu}$ it follows from the definition (7.12) of $\bar{A}$ that (see Figure 7.2 above)

$$
\mathfrak{A}_{\nu} \subset \bar{A} \text {. }
$$

This proves the density of the domain $D(\bar{A})$ in $C(\bar{\Omega})$, since the domain $D\left(\mathfrak{A}_{\nu}\right)$ is dense in $C(\bar{\Omega})$ (see assertion $(6.3)$ ).

(2) Now, let $(u, v)$ be an arbitrary element of the product space

$$
C(\bar{\Omega}) \oplus C(\bar{\Omega})
$$

such that there exists a sequence $\left\{u_{n}\right\} \subset D(\bar{A})$ which satisfies the conditions

$$
\begin{cases}u_{n} \longrightarrow u & \text { in } C(\bar{\Omega}), \\ A u_{n} \longrightarrow v & \text { in } C(\bar{\Omega}) .\end{cases}
$$

Then we have, by the boundedness of $G_{\alpha}^{0}$,

$$
G_{\alpha}^{0}\left(A u_{n}\right)=\alpha G_{\alpha}^{0} u_{n}-u_{n}+H_{\alpha}\left(\left.u_{n}\right|_{\partial \Omega}\right) \longrightarrow \alpha G_{\alpha}^{0} u-u+H_{\alpha}\left(\left.u\right|_{\partial \Omega}\right) \quad \text { in } C(\bar{\Omega}),
$$

and also

$$
G_{\alpha}^{0}\left(A u_{n}\right) \longrightarrow G_{\alpha}^{0} v \quad \text { in } C(\bar{\Omega}) .
$$

This proves that

$$
u=\alpha G_{\alpha}^{0} u+H_{\alpha}\left(\left.u\right|_{\partial \Omega}\right)-G_{\alpha}^{0} v \in W^{2, p}(\Omega) .
$$

Thus, by applying the operator $\alpha-A$ to the both hand sides of formula (7.13) we obtain that

$$
(\alpha-A) u=\alpha(\alpha-A) G_{\alpha}^{0} u-(\alpha-A) G_{\alpha}^{0} v=\alpha u-v
$$

so that

$$
A u=v \in C(\bar{\Omega}) .
$$

Summing up, we have proved that

$$
\left\{\begin{array}{l}
u \in D(\bar{A}), \\
\bar{A} u=v .
\end{array}\right.
$$

This proves the closedness of $\bar{A}$.

The proof of Lemma 7.1 is complete.

Remark 7.2 The domain $D(\bar{A})$ does not depend on $p$, for $N<p<\infty$ (see Section $9)$.

The (extended) operators $G_{\alpha}^{0}: C(\bar{\Omega}) \rightarrow C(\bar{\Omega})$ and $H_{\alpha}: C(\partial \Omega) \rightarrow C(\bar{\Omega})$ for $\alpha>0$ still satisfy formulas (7.2) and (7.3) respectively in the following sense (see [35, Lemma 10.12 and Corollary 10.13]):

Lemma 7.2 Let $\alpha>0$. Then we have the following assertions (i) and (ii). 
(i) For any $f \in C(\bar{\Omega})$, we have the formulas

$$
\left\{\begin{array}{l}
G_{\alpha}^{0} f \in D(\bar{A}), \\
(\alpha I-\bar{A}) G_{\alpha}^{0} f=f .
\end{array}\right.
$$

(ii) For any $\varphi \in C(\partial \Omega)$, we have the formulas

$$
\left\{\begin{array}{l}
H_{\alpha} \varphi \in D(\bar{A}), \\
(\alpha I-\bar{A}) H_{\alpha} \varphi=0 .
\end{array}\right.
$$

Proof Assertion (i): If $f \in C(\bar{\Omega})$, then it follows from the definition of $G_{\alpha}^{0}$ that

$$
\left\{\begin{array}{l}
G_{\alpha}^{0} f \in W^{2, p}(\Omega) \\
A\left(G_{\alpha}^{0} f\right)=\alpha G_{\alpha}^{0} f-f \in C(\bar{\Omega}) .
\end{array}\right.
$$

This proves the desired formulas (7.14).

Assertion (ii): If $\varphi \in C(\partial \Omega)$, we can find a sequence $\left\{\varphi_{j}\right\}$ in the space $C^{2}(\partial \Omega)$ such that

$$
\varphi_{j} \longrightarrow \varphi \quad \text { in } C(\partial \Omega) .
$$

Hence, we have, by the boundedness of $H_{\alpha}$,

$$
H_{\alpha} \varphi_{j} \longrightarrow H_{\alpha} \varphi \quad \text { in } C(\bar{\Omega})
$$

However, it follows that

$$
\begin{aligned}
& H_{\alpha} \varphi_{j} \in W^{2, p}(\Omega), \\
& A\left(H_{\alpha} \varphi_{j}\right)=\alpha H_{\alpha} \varphi_{j} \in C(\bar{\Omega}),
\end{aligned}
$$

so that

$$
H_{\alpha} \varphi_{j} \in D(\bar{A}) .
$$

Therefore, we have the assertions

$$
\left\{\begin{array}{l}
H_{\alpha} \varphi_{j} \in D(\bar{A}), \\
H_{\alpha} \varphi_{j} \longrightarrow H_{\alpha} \varphi \quad \text { in } C(\bar{\Omega}), \\
\bar{A}\left(H_{\alpha} \varphi_{j}\right) \longrightarrow \alpha H_{\alpha} \varphi \quad \text { in } C(\bar{\Omega}) .
\end{array}\right.
$$

This proves the desired formulas (7.15)

$$
\left\{\begin{array}{l}
H_{\alpha} \varphi \in D(\bar{A}), \\
\bar{A}\left(H_{\alpha} \varphi\right)=\alpha H_{\alpha} \varphi
\end{array}\right.
$$

since the operator $\bar{A}$ is closed.

The proof of Lemma 7.2 is complete.

Corollary 7.1 Every function $u \in D(\bar{A})$ can be written in the form

$$
u=G_{\alpha}^{0}((\alpha I-\bar{A}) u)+H_{\alpha}\left(\left.u\right|_{\partial \Omega}\right) \quad \text { for } \alpha>0 .
$$


Proof We let

$$
w=u-G_{\alpha}^{0}((\alpha I-\bar{A}) u)-H_{\alpha}\left(\left.u\right|_{\partial \Omega}\right) \in W^{2, p}(\Omega) .
$$

Then it follows from Lemma 7.2 that the function $w$ is in $D(\bar{A})$ and satisfies the conditions

$$
\begin{cases}(\alpha I-\bar{A}) w=0 & \text { in } \Omega, \\ w=0 & \text { on } \partial \Omega .\end{cases}
$$

Therefore, we can apply Theorem 4.1 to the operator $A-\alpha$ to obtain that

$$
0=w=u-G_{\alpha}^{0}((\alpha I-\bar{A}) u)-H_{\alpha}\left(\left.u\right|_{\partial \Omega}\right) .
$$

This proves the desired formula (7.16).

The proof of Corollary 7.1 is complete.

The situation of Lemma 7.2 can be visualized as in Figures 7.3 and 7.4 below.

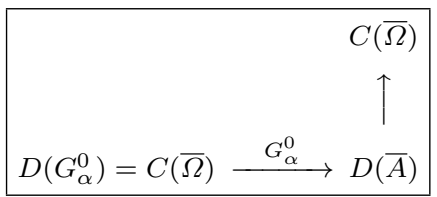

Fig. 7.3 The Green operator $G_{\alpha}^{0}$ and the domain $D(\bar{A})$ for formulas (7.14)

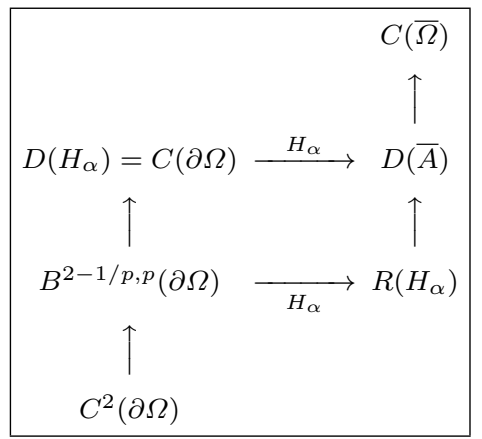

Fig. 7.4 The harmonic operator $H_{\alpha}$ and the domain $D(\bar{A})$ for formulas $(7.15)$

(II) Secondly, for $\alpha>0$ we introduce a linear trace operator

$$
\overline{L G_{\alpha}^{0}}: C(\bar{\Omega}) \longrightarrow C(\partial \Omega)
$$

as follows. 
(a) The domain $D\left(\overline{L G_{\alpha}^{0}}\right)$ of $\overline{L G_{\alpha}^{0}}$ is the space $C(\bar{\Omega})$.

(b) $\overline{L G_{\alpha}^{0}} f=L\left(G_{\alpha}^{0} f\right)=\mu\left(x^{\prime}\right) \frac{\partial}{\partial \mathbf{n}}\left(G_{\alpha}^{0} f\right)+\delta\left(x^{\prime}\right)\left(\left.f\right|_{\partial \Omega}\right)$ for every $f \in D\left(\overline{L G_{\alpha}^{0}}\right)$.

Here it should be emphasized that we have, by Sobolev's imbedding theorem (see [2, Theorem 4.12, Part II]),

$$
\left\{\begin{array}{l}
G_{\alpha}^{0} f \in W^{2, p}(\Omega) \subset C^{2-N / p}(\bar{\Omega}) \subset C^{1}(\bar{\Omega}), \\
A\left(G_{\alpha}^{0} f\right)=\alpha G_{\alpha}^{0} f-f \in C(\bar{\Omega}),
\end{array}\right.
$$

since $2-N / p>1$ for $N<p<\infty$.

Then we have the following lemma (see [35, Lemma 10.14]):

Lemma 7.3 The trace operators $\overline{L G_{\alpha}^{0}}: C(\bar{\Omega}) \rightarrow C(\partial \Omega)$ are non-negative and bounded for all $\alpha>0$.

Proof Let $f$ be an arbitrary function in $D\left(\overline{L G_{\alpha}^{0}}\right)=C(\bar{\Omega})$ such that $f(x) \geq 0$ on $\bar{\Omega}$. Then we have the assertions

$$
\begin{cases}G_{\alpha}^{0} f \in C^{1}(\bar{\Omega}), & \\ G_{\alpha}^{0} f \geq 0 & \text { on } \bar{\Omega} \\ G_{\alpha}^{0} f=0 & \text { on } \partial \Omega\end{cases}
$$

and so

$$
\begin{aligned}
\overline{L G_{\alpha}^{0}} f\left(x^{\prime}\right) & =L\left(G_{\alpha}^{0} f\right)\left(x^{\prime}\right)=\mu\left(x^{\prime}\right) \frac{\partial}{\partial \mathbf{n}}\left(G_{\alpha}^{0} f\right)\left(x^{\prime}\right)+\delta\left(x^{\prime}\right) f\left(x^{\prime}\right) \\
& \geq 0 \quad \text { on } \partial \Omega
\end{aligned}
$$

This proves that the operator $\overline{L G_{\alpha}^{0}}$ is non-negative.

By the non-negativity of $\overline{L G_{\alpha}^{0}}$, we have, for all $f \in D\left(\overline{L G_{\alpha}^{0}}\right)$,

$$
-\overline{L G_{\alpha}^{0}}\|f\|_{C(\bar{\Omega})} \leq \overline{L G_{\alpha}^{0}} f \leq \overline{L G_{\alpha}^{0}}\|f\|_{C(\bar{\Omega})} \quad \text { on } \partial \Omega
$$

This implies the boundedness of $\overline{L G_{\alpha}^{0}}$ with norm

$$
\left\|\overline{L G_{\alpha}^{0}}\right\|=\left\|L\left(G_{\alpha}^{0} 1\right)\right\|_{C(\partial \Omega)} .
$$

The proof of Lemma 7.3 is complete.

The trace operators $\overline{L G_{\alpha}^{0}}$ for $\alpha>0$ can be visualized as in Figures 7.5 and 7.6 below.

$$
\overline{L G_{\alpha}^{0}}: D\left(\overline{L G_{\alpha}^{0}}\right)=C(\bar{\Omega}) \stackrel{G_{\alpha}^{0}}{\longrightarrow} D(\bar{A}) \stackrel{L}{\longrightarrow} C(\partial \Omega) .
$$

Fig. 7.5 The mapping property of the operators $\overline{L G_{\alpha}^{0}}$ in Lemma 7.3 
Remark 7.3 More generally, we can prove that the trace operators

$$
\begin{aligned}
\widetilde{L G_{\alpha}^{0}}: L^{\infty}(\Omega) & \longrightarrow C(\partial \Omega) \\
f & \longmapsto L\left(\widetilde{G_{\alpha}^{0}} f\right)
\end{aligned}
$$

are non-negative and bounded for all $\alpha>0$, with norm (see Figure 7.6 below)

$$
\left\|\widetilde{L G_{\alpha}^{0}}\right\|=\left\|L\left(G_{\alpha}^{0} 1\right)\right\|_{C(\partial \Omega)}=\left\|\overline{L G_{\alpha}^{0}}\right\| .
$$

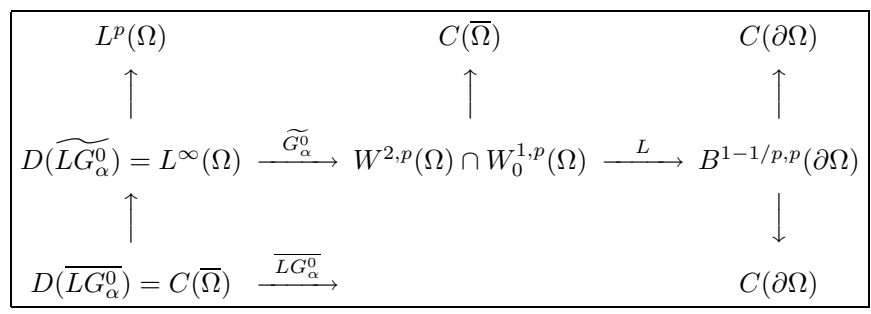

Fig. 7.6 The mapping properties of the operators $\overline{L G_{\alpha}^{0}}$ and $\widetilde{L G_{\alpha}^{0}}$

The next lemma states a fundamental relationship between the trace operators $\overline{L G_{\alpha}^{0}}$ and $\overline{L G_{\beta}^{0}}$ for all $\alpha, \beta>0$ (see [35, Lemma 10.15]):

Lemma 7.4 For any $\alpha, \beta>0$, we have the equation

$$
\overline{L G_{\alpha}^{0}} f-\overline{L G_{\beta}^{0}} f+(\alpha-\beta) \overline{L G_{\alpha}^{0}}\left(G_{\beta}^{0} f\right)=0 \quad \text { for each } f \in C(\bar{\Omega}) .
$$

Proof In fact, since the function

$$
u:=G_{\alpha}^{0} f-G_{\beta}^{0} f+(\alpha-\beta) G_{\alpha}^{0}\left(G_{\beta}^{0} f\right) \in W^{2, p}(\Omega) \cap W_{0}^{1, p}(\Omega)
$$

is a unique solution of the Dirichlet problem

$$
\begin{cases}(\alpha-A) u=0 & \text { almost everywhere in } \Omega, \\ u=0 & \text { on } \partial \Omega,\end{cases}
$$

it follows from an application of Theorem 4.1 with $A:=A-\alpha$ that

$$
G_{\alpha}^{0} f-G_{\beta}^{0} f+(\alpha-\beta) G_{\alpha}^{0}\left(G_{\beta}^{0} f\right)=0 .
$$

Therefore, the desired formula (7.17) follows by applying the boundary operator $L$ to the both sides of formula (7.18).

The proof of Lemma 7.4 is complete.

(III) Finally, by virtue of assertion (7.5) we can introduce a linear operator

$$
L H_{\alpha}: C(\partial \Omega) \longrightarrow C(\partial \Omega)
$$

as follows. 
(c) The domain $D\left(L H_{\alpha}\right)$ of $L H_{\alpha}$ is the space $B^{2-1 / p, p}(\partial \Omega)$ for $N<p<\infty$.

(d) $L H_{\alpha} \psi=L\left(H_{\alpha} \psi\right)=\mu\left(x^{\prime}\right) \frac{\partial}{\partial \mathbf{n}}\left(H_{\alpha} \psi\right)+\beta\left(x^{\prime}\right) \cdot \partial_{x^{\prime}} \psi+\gamma\left(x^{\prime}\right) \psi-\alpha \delta\left(x^{\prime}\right) \psi$ for every $\psi \in D\left(L H_{\alpha}\right)$.

By assertion (7.5), we remark that the domain $D\left(L H_{\alpha}\right)$ is dense in the space $C(\partial \Omega)$ :

$$
C^{1}(\partial \Omega) \subset D\left(L H_{\alpha}\right)=B^{1-1 / p, p}(\partial \Omega) \subset C(\partial \Omega) \text { for } N<p<\infty .
$$

Then we have the following lemma (see [35, Lemma 10.16]):

Lemma 7.5 The operator $L H_{\alpha}$ for $\alpha>0$ has its minimal closed extension $\overline{L H_{\alpha}}$ in the space $C(\partial \Omega)$.

Proof We apply part (i) of the Hille-Yosida-Ray theorem (Theorem 3.2) with

$$
K:=\partial \Omega, \quad B:=L H_{\alpha}
$$

To do this, it suffices to show that the operator $L H_{\alpha}$ satisfies condition $(\beta)$ with $K:=K_{0}=\partial \Omega$ of the same theorem.

Assume that a function $\varphi$ in the domain $D\left(L H_{\alpha}\right)=B^{2-1 / p, p}(\partial \Omega)$ takes its positive maximum at some point $x_{0}^{\prime}$ of $\partial \Omega$. Since the harmonic function

$$
H_{\alpha} \varphi \in W^{2, p}(\Omega)
$$

satisfies the conditions

$$
\begin{cases}(A-\alpha) H_{\alpha} \varphi=0 & \text { in } \Omega, \\ H_{\alpha} \varphi=\varphi & \text { on } \partial \Omega,\end{cases}
$$

by applying the weak maximum principle (see [6], [41, p. 191, Lemma 3.25], [36, Theorem 8.1]) with $A:=A-\alpha$ to the function $H_{\alpha} \psi$, we find that the function $H_{\alpha} \varphi$ takes its positive maximum at a boundary point $x_{0}^{\prime} \in \partial \Omega$ :

$$
\max _{x \in \bar{\Omega}} H_{\alpha} \varphi(x)=H_{\alpha} \varphi\left(x_{0}^{\prime}\right)=\varphi\left(x_{0}^{\prime}\right)>0 .
$$

Thus we can apply Hopf's boundary point lemma (see [6], [41, p. 192, Lemma $3.26]$, [37, Lemma 6.1]) to obtain that

$$
\frac{\partial}{\partial \mathbf{n}}\left(H_{\alpha} \varphi\right)\left(x_{0}^{\prime}\right)<0 .
$$

However, it should be noticed that the coefficients of the boundary condition $L$ satisfy the conditions

$$
\begin{cases}\mu\left(x^{\prime}\right)>0 & \text { on } \partial \Omega \\ \gamma\left(x^{\prime}\right) \leq 0 & \text { on } \partial \Omega \\ \delta\left(x^{\prime}\right) \geq 0 & \text { on } \partial \Omega\end{cases}
$$

Hence we have the inequality

$$
L H_{\alpha} \varphi\left(x_{0}^{\prime}\right)=\mu\left(x_{0}^{\prime}\right) \frac{\partial}{\partial \mathbf{n}}\left(H_{\alpha} \varphi\right)\left(x_{0}^{\prime}\right)+\beta\left(x_{0}^{\prime}\right) \cdot \partial_{x^{\prime}} \varphi\left(x_{0}^{\prime}\right)+\gamma\left(x_{0}^{\prime}\right) \varphi\left(x_{0}^{\prime}\right)-\alpha \delta\left(x_{0}^{\prime}\right) \varphi\left(x_{0}^{\prime}\right)
$$




$$
\begin{aligned}
& =\mu\left(x_{0}^{\prime}\right) \frac{\partial}{\partial \mathbf{n}}\left(H_{\alpha} \varphi\right)\left(x_{0}^{\prime}\right)+\gamma\left(x_{0}^{\prime}\right) \varphi\left(x_{0}^{\prime}\right)-\alpha \delta\left(x_{0}^{\prime}\right) \varphi\left(x_{0}^{\prime}\right) \\
& \leq \mu\left(x_{0}^{\prime}\right) \frac{\partial}{\partial \mathbf{n}}\left(H_{\alpha} \varphi\right)\left(x_{0}^{\prime}\right)<0 .
\end{aligned}
$$

This verifies condition $(\beta)$ of Theorem 3.2.

The proof of Lemma 7.5 is complete.

Remark 7.4 The closed operator $\overline{L H_{\alpha}}$ enjoys the positive maximum principle:

If a function $\varphi$ in the domain $D\left(\overline{L H_{\alpha}}\right)$ takes its positive maximum at some point $x_{0}^{\prime}$ of $\partial \Omega$, then we have the inequality

$$
\overline{L H_{\alpha}} \varphi\left(x_{0}^{\prime}\right) \leq 0 \text {. }
$$

Namely, this assertion implies that the closed operator $\overline{L H_{\alpha}}$ satisfies condition $\left(\beta^{\prime}\right)$ in Theorem 3.2.

The next lemma states a fundamental relationship between the operators $\overline{L H_{\alpha}}$ and $\overline{L H_{\beta}}$ for all $\alpha, \beta>0$ (see [35, Lemma 10.18]):

Lemma 7.6 The domain $D\left(\overline{L H_{\alpha}}\right)$ of $\overline{L H_{\alpha}}$ does not depend on $\alpha>0$; so we denote by $\mathcal{D}$ the common domain. Then we have, for all $\alpha, \beta>0$,

$$
\overline{L H_{\alpha}} \varphi-\overline{L H_{\beta}} \varphi+(\alpha-\beta) \overline{L G_{\alpha}^{0}}\left(H_{\beta} \varphi\right)=0 \quad \text { for each } \varphi \in \mathcal{D} .
$$

Proof Let $\varphi$ be an arbitrary function in $D\left(\overline{L H_{\beta}}\right)$, and choose a sequence $\left\{\varphi_{j}\right\}$ in $D\left(L H_{\beta}\right)=B^{2-1 / p, p}(\partial \Omega)$ such that

$$
\begin{cases}\varphi_{j} \longrightarrow \varphi & \text { in } C(\partial \Omega), \\ L H_{\beta} \varphi_{j} \longrightarrow \overline{L H_{\beta}} \varphi & \text { in } C(\partial \Omega) .\end{cases}
$$

Then it follows from the boundedness of the operators $H_{\beta}$ and $\overline{L G_{\alpha}^{0}}$ that

$$
\overline{L G_{\alpha}^{0}}\left(H_{\beta} \varphi_{j}\right) \longrightarrow \overline{L G_{\alpha}^{0}}\left(H_{\beta} \varphi\right) \text { in } C(\partial \Omega) .
$$

Therefore, by using formula (7.10) with $\varphi:=\varphi_{j}$ we obtain that

$$
\begin{aligned}
L H_{\alpha} \varphi_{j} & =L H_{\beta} \varphi_{j}-(\alpha-\beta) \overline{L G_{\alpha}^{0}}\left(H_{\beta} \varphi_{j}\right) \\
& \longrightarrow \overline{L H_{\beta}} \varphi-(\alpha-\beta) \overline{L G_{\alpha}^{0}}\left(H_{\beta} \varphi\right) \quad \text { in } C(\partial \Omega) .
\end{aligned}
$$

Since the operator $\overline{L H_{\alpha}}$ is closed, it follows that

$$
\left\{\begin{array}{l}
\varphi \in D\left(\overline{L H_{\alpha}}\right), \\
\overline{L H_{\alpha}} \varphi=\overline{L H_{\beta}} \varphi-(\alpha-\beta) \overline{L G_{\alpha}^{0}}\left(H_{\beta} \varphi\right) .
\end{array}\right.
$$

This proves the desired equation (7.19).

Conversely, we have, by interchanging $\alpha$ and $\beta$,

$$
D\left(\overline{L H_{\alpha}}\right) \subset D\left(\overline{L H_{\beta}}\right),
$$

and so

$$
D\left(\overline{L H_{\alpha}}\right)=D\left(\overline{L H_{\beta}}\right) \quad \text { for all } \alpha, \beta>0 .
$$

The proof of Lemma 7.6 is complete. 
Remark 7.5 It should be noticed that the common domain $\mathcal{D}$ contains the Besov space $B^{2-1 / p, p}(\partial \Omega)$, since we have the formula

$$
B^{2-1 / p, p}(\partial \Omega)=D\left(L H_{\alpha}\right) \subset D\left(\overline{L H_{\alpha}}\right)=\mathcal{D} .
$$

The operators $L H_{\alpha}$ and $\overline{L H_{\alpha}}$ for $\alpha>0$ can be visualized as in Figure 7.7 below.

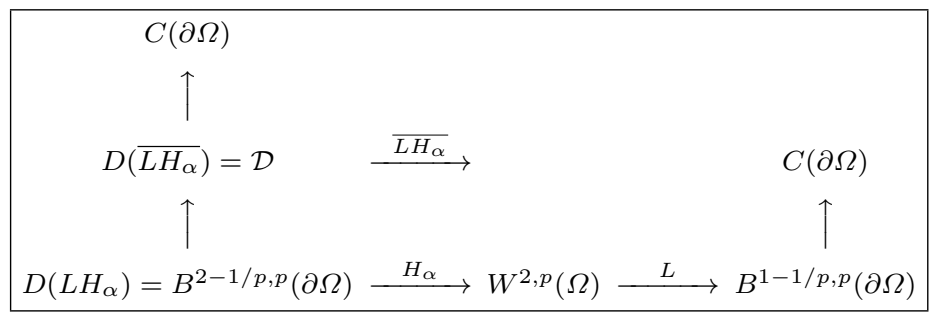

Fig. 7.7 The mapping properties of the operators $L H_{\alpha}$ and $\overline{L H_{\alpha}}$ in Lemmas 7.5 and 7.6

\subsection{General Existence Theorem for Feller Semigroups}

Now we can give a general existence theorem for Feller semigroups on the boundary $\partial \Omega$ in terms of the boundary value problem (7.20), which is a generalization of the classical Fredholm integral equation. The next theorem asserts that the operator $\overline{L H_{\alpha}}$ is the infinitesimal generator of some Feller semigroup on the state space $\partial \Omega$ if and only if problem (7.20) is solvable for sufficiently many functions $\varphi$ in the Banach space $C(\partial \Omega)$ (see [35, Theorem 10.19]):

Theorem 7.2 (i) If the closed operator $\overline{L H_{\alpha}}$ for $\alpha>0$ is the infinitesimal generator of a Feller semigroup on the state space $\partial \Omega$, then, for each constant $\lambda>0$ the Wentzell boundary value problem

$$
\begin{cases}(\alpha-A) u=0 & \text { in } \Omega, \\ (\lambda-L) u=\varphi & \text { on } \partial \Omega\end{cases}
$$

has a solution $u \in W^{2, p}(\Omega)$ for any $\varphi$ in some dense subset of the Banach space $C(\partial \Omega)$.

(ii) Conversely, if the Wentzell boundary value problem (7.20) has a solution $u \in$ $W^{2, p}(\Omega)$ for any $\varphi$ in some dense subset of $C(\partial \Omega)$ for a constant $\lambda \geq 0$, then the closed operator $\overline{L H_{\alpha}}$ is the infinitesimal generator of some Feller semigroup on the state space $\partial \Omega$.

Proof (i) If the operator $\overline{L H_{\alpha}}$ generates a Feller semigroup on the state space $\partial \Omega$, by applying part (i) of the Hille-Yosida theorem (Theorem 3.1) with $K:=\partial \Omega$ to the operator $\mathfrak{A}:=\overline{L H_{\alpha}}$ we obtain that

$$
R\left(\lambda I-\overline{L H_{\alpha}}\right)=C(\partial \Omega) \text { for each } \lambda>0 .
$$


This implies that the range $R\left(\lambda I-L H_{\alpha}\right)$ is a dense subset of $C(\partial \Omega)$ for each $\lambda>0$. However, if $\varphi \in R\left(\lambda I-L H_{\alpha}\right)$ with $\varphi=\left(\lambda I-L H_{\alpha}\right) \psi$ for some $\psi \in B^{2-1 / p, p}(\partial \Omega)$, then the harmonic function $u=H_{\alpha} \psi \in W^{2, p}(\Omega)$ is a solution of the Wentzell boundary value problem (7.20). This proves part (i).

(ii) We apply part (ii) of the Hille-Yosida-Ray theorem (Theorem 3.2) with $K:=\partial \Omega$ to the operator $L H_{\alpha}$. To do this, it suffices to show that the operator $L H_{\alpha}$ satisfies condition $(\gamma)$ of the same theorem, since it satisfies condition $\left(\beta^{\prime}\right)$, as is shown in the proof of Lemma 7.5.

By the uniqueness theorem for the Dirichlet problem (7.1), it follows that any function $u \in W^{2, p}(\Omega)$ which satisfies the equation

$$
(\alpha-A) u=0 \quad \text { in } \Omega
$$

can be written in the form

$$
\left\{\begin{array}{l}
u=H_{\alpha}\left(\left.u\right|_{\partial \Omega}\right), \\
\left.u\right|_{\partial \Omega} \in B^{2-1 / p, p}(\partial \Omega)=D\left(L H_{\alpha}\right) \subset D\left(\overline{L H_{\alpha}}\right)=\mathcal{D} .
\end{array}\right.
$$

Thus we find that if there exists a solution $u \in W^{2, p}(\Omega)$ of the Wentzell boundary value problem (7.20) for some function $\varphi \in C(\partial \Omega)$, then we have the formula

$$
\left(\lambda I-L H_{\alpha}\right)\left(\left.u\right|_{\partial \Omega}\right)=\varphi,
$$

and so

$$
\varphi \in R\left(\lambda I-L H_{\alpha}\right) \text {. }
$$

Therefore, if there exists a constant $\lambda \geq 0$ such that the Wentzell boundary value problem (7.20) has a solution $u \in W^{2, p}(\Omega)$ for any $\varphi$ in some dense subset of $C(\partial \Omega)$, then the range $R\left(\lambda I-L H_{\alpha}\right)$ is dense in the space $C(\partial \Omega)$ :

$$
R\left(\lambda I-\overline{L H_{\alpha}}\right)=C(\partial \Omega) \text { for some } \lambda \geq 0 .
$$

This verifies condition $(\gamma)$ (with $\alpha_{0}:=\lambda$ ) of Theorem 3.2.

Hence, part (ii) follows from an application of Theorem 3.2.

The proof of Theorem 7.2 is complete.

We conclude this subsection by giving a precise meaning to the boundary conditions $L u$ for functions $u$ in the domain $D(\bar{A})$ given by the definition (7.12).

We let

$$
D(L):=\left\{u \in D(\bar{A}):\left.u\right|_{\partial \Omega} \in \mathcal{D}\right\}=\left\{u \in W^{2, p}(\Omega): A u \in C(\bar{\Omega}),\left.u\right|_{\partial \Omega} \in \mathcal{D}\right\},
$$

where $\mathcal{D}$ is the common domain of the operators $\overline{L H_{\alpha}}$ for all $\alpha>0$ (see Lemma 7.6). Then Corollary 7.1 asserts that every function $u$ in $D(L) \subset D(\bar{A})$ can be written in the form

$$
u=G_{\alpha}^{0}((\alpha I-\bar{A}) u)+H_{\alpha}\left(\left.u\right|_{\partial \Omega}\right) \quad \text { for all } \alpha>0 .
$$

Hence we can define the boundary condition $L u$ by the formula

$$
L u:=\overline{L G_{\alpha}^{0}}((\alpha I-\bar{A}) u)+\overline{L H_{\alpha}}\left(\left.u\right|_{\partial \Omega}\right) .
$$

The next lemma justifies the definition (7.21) of $L u$ for $u \in D(L)$ (see [35, Lemma 10.20]): 
Lemma 7.7 The right-hand side of formula (7.21) depends only on $u$, not on the choice of expression (7.16).

Proof Assume that

$$
u=G_{\alpha}^{0}((\alpha I-\bar{A}) u)+H_{\alpha}\left(\left.u\right|_{\partial \Omega}\right)=G_{\beta}^{0}((\beta I-\bar{A}) u)+H_{\beta}\left(\left.u\right|_{\partial \Omega}\right),
$$

where $\alpha>0$ and $\beta>0$. Then it follows from formula (7.17) with $f:=(\alpha I-\bar{A}) u$ and formula (7.19) with $\psi:=\left.u\right|_{\partial \Omega}$ that

$$
\begin{aligned}
& \overline{L G_{\alpha}^{0}}((\alpha I-\bar{A}) u)+\overline{L H_{\alpha}}\left(\left.u\right|_{\partial \Omega}\right) \\
= & \overline{L G_{\beta}^{0}}((\alpha I-\bar{A}) u)-(\alpha-\beta) \overline{L G_{\alpha}^{0}} G_{\beta}^{0}((\alpha I-\bar{A}) u) \\
& +\overline{L H_{\beta}}\left(\left.u\right|_{\partial \Omega}\right)-(\alpha-\beta) \overline{L G_{\alpha}^{0}} H_{\beta}\left(\left.u\right|_{\partial \Omega}\right) \\
= & \overline{L G_{\beta}^{0}}((\beta I-A) u)+\overline{L H_{\beta}}\left(\left.u\right|_{\partial \Omega}\right) \\
& +(\alpha-\beta)\left\{\overline{L G_{\beta}^{0}} u-\overline{L G_{\alpha}^{0}} G_{\beta}^{0}(\alpha I-\bar{A}) u-\overline{L G_{\alpha}^{0}} H_{\beta}\left(\left.u\right|_{\partial \Omega}\right)\right\} .
\end{aligned}
$$

However, the last term of formula (7.22) vanishes. Indeed, it follows from formula (7.16) with $\alpha:=\beta$ and formula (7.17) with $f:=u$ that

$$
\begin{aligned}
& \overline{L G_{\beta}^{0}} u-\overline{L G_{\alpha}^{0}}\left(G_{\beta}^{0}(\alpha I-\bar{A}) u\right)-\overline{L G_{\alpha}^{0}} H_{\beta}\left(\left.u\right|_{\partial \Omega}\right) \\
= & \overline{L G_{\beta}^{0}} u-\overline{L G_{\alpha}^{0}}\left(G_{\beta}^{0}(\beta I-\bar{A}) u+H_{\beta}\left(\left.u\right|_{\partial \Omega}\right)+(\alpha-\beta) G_{\beta}^{0} u\right) \\
= & \overline{L G_{\beta}^{0}} u-\overline{L G_{\alpha}^{0}} u-(\alpha-\beta) \overline{L G_{\alpha}^{0}} G_{\beta}^{0} u \\
= & 0 .
\end{aligned}
$$

Therefore, we obtain from formula (7.22) that

$$
\overline{L G_{\alpha}^{0}}((\alpha I-\bar{A}) u)+\overline{L H_{\alpha}}\left(\left.u\right|_{\partial \Omega}\right)=\overline{L G_{\beta}^{0}}((\beta I-\bar{A}) u)+\overline{L H_{\beta}}\left(\left.u\right|_{\partial \Omega}\right) .
$$

The proof of Lemma 7.7 is complete.

\section{Proof of Theorem 1.1}

This section is devoted to the proof of Theorem 1.1. The crucial point in the proof is that we consider the term

$$
\delta\left(x^{\prime}\right)\left(\left.A u\right|_{\partial \Omega}\right)
$$

of sticking (or viscosity) phenomenon in the original Wentzell boundary condition

$$
L u=L_{\nu} u-\delta\left(x^{\prime}\right)\left(\left.A u\right|_{\partial \Omega}\right) \quad \text { on } \partial \Omega
$$

as a term of perturbation of the oblique derivative boundary condition

$$
L_{\nu} u=\mu\left(x^{\prime}\right) \frac{\partial u}{\partial \mathbf{n}}+\beta\left(x^{\prime}\right) \cdot \partial_{x^{\prime}} u+\gamma\left(x^{\prime}\right) u \quad \text { on } \partial \Omega .
$$

More precisely, we make use of a generation theorem for Feller semigroups with oblique derivative boundary condition $L_{\nu}$ to verify all the conditions of the HilleYosida-Ray theorem (Theorem 3.2) for the operator $\mathfrak{A}$ defined by formula (1.4), just as in the proof of [35, Theorem 10.21].

We shall apply part (ii) of Theorem 3.2 to the operator $\mathfrak{A}$. The proof is carried out in a series of lemmas and propositions.

Step (I): First, we prove the following lemma: 
Lemma 8.1 If condition (H.1) is satisfied, then the closed operator $\overline{L_{\nu} H_{\alpha}}$ is the generator of some Feller semigroup on the state space $\partial \Omega$ for any $\alpha>0$.

Proof We apply Theorem 7.2 with $L:=L_{\nu}$. By virtue of Theorem 5.1 with $f:=0$, we obtain that the oblique derivative problem

$$
\begin{cases}(A-\alpha) u=0 & \text { in } \Omega, \\ L_{\nu} u=\varphi & \text { on } \partial \Omega\end{cases}
$$

has a unique function $u \in W^{2, p}(\Omega)$ for any function $\varphi \in B^{1-1 / p, p}(\partial \Omega)$, if $N<p<$ $\infty$. Here it should be emphasized that we have, by assertion (7.5),

$$
C^{1}(\partial \Omega) \subset B^{1-1 / p, p}(\partial \Omega) \subset C(\partial \Omega) \text { for } N<p<\infty .
$$

Hence, for any $\varphi \in B^{1-1 / p, p}(\partial \Omega)$ we can find a function $\psi \in B^{2-1 / p, p}(\partial \Omega)$ such that the harmonic function

$$
u=H_{\alpha} \psi \in W^{2, p}(\Omega)
$$

satisfies the boundary condition

$$
L_{\nu} H_{\alpha} \psi=L_{\nu}\left(H_{\alpha} \psi\right)=\mu\left(x^{\prime}\right) \frac{\partial\left(H_{\alpha} \psi\right)}{\partial \mathbf{n}}+\beta\left(x^{\prime}\right) \cdot \partial_{x^{\prime}} \psi+\gamma\left(x^{\prime}\right) \psi=\varphi \quad \text { on } \partial \Omega .
$$

This implies that the range $R\left(L_{\nu} H_{\alpha}\right)$ is a dense subset of the space $C(\partial \Omega)$.

Therefore, by applying part (ii) of Theorem 7.2 with $\lambda:=0$ we obtain that the closed operator $\overline{L_{\nu} H_{\alpha}}$ generates a Feller semigroup on the state space $\partial \Omega$, for any $\alpha>0$.

The proof of Lemma 8.1 is complete.

Step (II): Secondly, we prove the following lemma:

Lemma 8.2 If condition (H.1) is satisfied, then the closed operator $\overline{L_{\alpha}}$ generates a Feller semigroup on the state space $\partial \Omega$ for any $\alpha>0$.

Proof We apply Corollary 3.2 with $K:=\partial \Omega$ to the operator $\overline{L H_{\alpha}}$ for each $\alpha>0$.

By formula (1.2), we find that the operator $\overline{L H_{\alpha}}$ can be written in the form

$$
\overline{L H_{\alpha}}=\overline{L_{\nu} H_{\alpha}}+M,
$$

where

$$
M:=-\alpha \delta\left(x^{\prime}\right)
$$

is a bounded linear operator on $C(\partial \Omega)$ into itself. However, since $\delta\left(x^{\prime}\right) \geq 0$ on $\partial \Omega$, it follows that the bounded operator $M$ satisfies condition $\left(\beta^{\prime}\right)$ of the HilleYosida-Ray theorem (Theorem 3.2):

$\left(\beta^{\prime}\right)$ If $\psi \in C(\partial \Omega)$ takes a positive maximum at a point $x_{0}^{\prime}$ of $\partial \Omega$, then we have the inequality

$$
M \psi\left(x_{0}^{\prime}\right)=-\alpha \delta\left(x_{0}^{\prime}\right) \psi\left(x_{0}^{\prime}\right) \leq 0 .
$$


By virtue of Lemma 8.1, we can apply Corollary 3.2 with

$$
K:=\partial \Omega, \quad A:=\overline{L_{\nu} H_{\alpha}}, \quad M:=-\alpha \delta\left(x^{\prime}\right)
$$

to obtain that the closed operator $\overline{L H_{\alpha}}=\overline{L_{\nu} H_{\alpha}}+M$ generates a Feller semigroup on the state space $\partial \Omega$ for any $\alpha>0$.

The proof of Lemma 8.2 is complete.

Step (III): Thirdly, we prove the following lemma:

Lemma 8.3 Assume that condition (H.1) is satisfied. Then the equation

$$
\overline{L H_{\alpha}} \psi=\varphi
$$

has a unique solution $\psi$ in $D\left(\overline{L H_{\alpha}}\right)$ for any $\varphi \in C(\partial \Omega)$; hence the inverse ${\overline{L H_{\alpha}}}^{-1}$ of $\overline{L H_{\alpha}}$ can be defined on the whole space $C(\partial \Omega)$. Furthermore, the operator $-{\overline{L H_{\alpha}}}^{-1}$ is non-negative and bounded on the space $C(\partial \Omega)$.

Proof Since the function $H_{\alpha} 1$ takes its positive maximum 1 only on the boundary $\partial \Omega$, we can apply Hopf's boundary point lemma (see [6], [41, p. 192, Lemma 3.26], [37, Lemma 6.1]) to obtain that

$$
\frac{\partial}{\partial \mathbf{n}}\left(H_{\alpha} 1\right)<0 \quad \text { on } \partial \Omega
$$

Hence it follows from inequality (8.2) and condition (H.1) that

$$
\begin{aligned}
L H_{\alpha} 1\left(x^{\prime}\right) & =L\left(H_{\alpha} 1\right)\left(x^{\prime}\right)=\mu\left(x^{\prime}\right) \frac{\partial}{\partial \mathbf{n}}\left(H_{\alpha} 1\right)\left(x^{\prime}\right)+\gamma\left(x^{\prime}\right)-\alpha \delta\left(x^{\prime}\right) \\
& \leq \mu\left(x^{\prime}\right) \frac{\partial}{\partial \mathbf{n}}\left(H_{\alpha} 1\right)\left(x^{\prime}\right)<0 \quad \text { on } \partial \Omega,
\end{aligned}
$$

so that

$$
\ell_{\alpha}=-\sup _{x^{\prime} \in \partial \Omega} L H_{\alpha} 1\left(x^{\prime}\right)>0 .
$$

Furthermore, by applying Corollary 3.1 with

$$
K:=\partial \Omega, \quad A:=\overline{L H_{\alpha}}, \quad c:=\ell_{\alpha},
$$

we obtain that the operator $\overline{L H_{\alpha}}+\ell_{\alpha} I$ is the infinitesimal generator of some Feller semigroup on the state space $\partial \Omega$.

Therefore, since $\ell_{\alpha}>0$, it follows from an application of part (i) of the HilleYosida theorem (Theorem 3.1) with $\mathfrak{A}:=\overline{L H_{\alpha}}+\ell_{\alpha} I$ that the equation

$$
-\overline{L H_{\alpha}} \psi=\left(\ell_{\alpha} I-\left(\overline{L H_{\alpha}}+\ell_{\alpha} I\right)\right) \psi=\varphi
$$

has a unique solution $\psi \in D\left(\overline{L H_{\alpha}}\right)$ for any $\varphi \in C(\partial \Omega)$, and further that the operator

$$
-{\overline{L H_{\alpha}}}^{-1}=\left(\ell_{\alpha} I-\left(\overline{L H_{\alpha}}+\ell_{\alpha} I\right)\right)^{-1}
$$

is non-negative and bounded on the space $C(\partial \Omega)$ with norm

$$
\left\|-{\overline{L H_{\alpha}}}^{-1}\right\|=\left\|\left(\ell_{\alpha} I-\left(\overline{L H_{\alpha}}+\ell_{\alpha} I\right)\right)^{-1}\right\| \leq \frac{1}{\ell_{\alpha}} .
$$

The proof of Lemma 8.3 is complete. 
Step (IV): By assertion (8.1), we can define the Green operator $G_{\alpha}$ for $\alpha>0$ by the formula

$$
G_{\alpha} f=G_{\alpha}^{0} f-H_{\alpha}\left({\overline{L H_{\alpha}}}^{-1}\left(\overline{L G_{\alpha}^{0}} f\right)\right) \text { for } f \in C(\bar{\Omega}) .
$$

Namely, the Green operator $G_{\alpha}$ can be expressed in the matrix form via the Boutet de Monvel calculus (see [35, p. 349, Section 7.7] and Figure 8.1 below):

$$
\left(\begin{array}{cc}
G_{\alpha}^{0} & H_{\alpha} \\
& \\
\overline{L G_{\alpha}^{0}} & -{\overline{L H_{\alpha}}}^{-1}
\end{array}\right): \underset{C(\partial \Omega)}{\bigoplus_{C(\bar{\Omega})}} \longrightarrow \underset{\bigoplus}{\bigoplus}
$$

$$
\begin{array}{ccc}
D\left(G_{\alpha}^{0}\right)=C(\bar{\Omega}) & \stackrel{\overline{L G_{\alpha}^{0}}}{\longrightarrow} \quad C(\partial \Omega) \\
G_{\alpha}^{0} \downarrow & -\overline{L H_{\alpha}}-1 \downarrow \\
D(\bar{A}) & \longleftarrow H_{\alpha} & \mathcal{D}=D\left(\overline{L H_{\alpha}}\right)
\end{array}
$$

Fig. 8.1 The mapping property of each term in formula (8.3) via the Boutet de Monvel calculus

Now we are in a position to prove the following proposition:

Proposition 8.1 Assume that condition (H.1) is satisfied. Then we have the formula

$$
G_{\alpha}=(\alpha I-\mathfrak{A})^{-1} \quad \text { for all } \alpha>0,
$$

where $\mathfrak{A}$ is a linear operator from $C(\bar{\Omega})$ into itself defined as follows:

(a) The domain $D(\mathfrak{A})$ is the set

$$
D(\mathfrak{A})=\left\{u \in D(\bar{A}):\left.u\right|_{\partial \Omega} \in \mathcal{D}, L u=0 \text { on } \partial \Omega\right\} .
$$

(b) $\mathfrak{A} u=\bar{A} u=A u$ for every $u \in D(\mathfrak{A})$.

Here $\mathcal{D}$ is the common domain of the operators $\overline{L H_{\alpha}}$ for all $\alpha>0$ (see Lemma 7.6).

Remark 8.1 By the definition (7.12) of $\bar{A}$, it follows that the domain $D(\mathfrak{A})$ in Proposition 8.1 coincides with that of the definition (1.4).

Proof The proof of Proposition 8.1 is divided into two steps.

(1) In view of Lemmas 7.2 and 7.6, it follows that we have, for every $f \in C(\bar{\Omega})$,

$$
\left\{\begin{array}{l}
G_{\alpha} f=G_{\alpha}^{0} f-H_{\alpha}\left({\overline{L H_{\alpha}}}^{-1}\left(\overline{L G_{\alpha}^{0}} f\right)\right) \in D(\bar{A}) \\
\left.\left(G_{\alpha} f\right)\right|_{\partial \Omega}=-{\overline{L H_{\alpha}}}^{-1}\left(\overline{L G_{\alpha}^{0}} f\right) \in D\left(\overline{L H_{\alpha}}\right)=\mathcal{D} \\
L G_{\alpha} f=\overline{L G_{\alpha}^{0}} f-\overline{L H_{\alpha}}\left({\overline{L H_{\alpha}}}^{-1}\left(\overline{L G_{\alpha}^{0}} f\right)\right)=0 \text { on } \partial \Omega
\end{array}\right.
$$

and

$$
(\alpha I-\bar{A}) G_{\alpha} f=f .
$$


That is, we have the assertions

$$
\left\{\begin{array}{l}
G_{\alpha} f \in D(\mathfrak{A}), \\
(\alpha I-\mathfrak{A}) G_{\alpha} f=f .
\end{array}\right.
$$

This proves that

$$
(\alpha I-\mathfrak{A}) G_{\alpha}=I \quad \text { on } C(\bar{\Omega}) .
$$

(2) In order to prove the desired formula (8.4), it suffices to show the injectivity of the operator $\alpha I-\mathfrak{A}$ for $\alpha>0$.

Assume that

$$
u \in D(\mathfrak{A}) \quad \text { and } \quad(\alpha I-\mathfrak{A}) u=0 .
$$

Then, by Corollary 7.1 it follows that the function $u$ can be written as follows:

$$
u=H_{\alpha}\left(\left.u\right|_{\partial \Omega}\right),\left.\quad u\right|_{\partial \Omega} \in \mathcal{D}=D\left(\overline{L H_{\alpha}}\right) .
$$

Thus we have the assertion

$$
\overline{L H_{\alpha}}\left(\left.u\right|_{\partial \Omega}\right)=L u=0 \quad \text { on } \partial \Omega .
$$

In view of assertion (8.1), this implies that

$$
\left.u\right|_{\partial \Omega}=0,
$$

so that

$$
u=H_{\alpha}\left(\left.u\right|_{\partial \Omega}\right)=0 \quad \text { in } \Omega .
$$

The proof of Proposition 8.1 is complete.

Step (V): Moreover, we prove the following proposition:

Proposition 8.2 Let $\alpha>0$. If condition (H.1) is satisfied, then the Green operator $G_{\alpha}$ given by formula (8.3) is non-negative and bounded on the space $C(\bar{\Omega})$ with norm

$$
\left\|G_{\alpha}\right\| \leq \frac{1}{\alpha} \quad \text { for all } \alpha>0 .
$$

Proof (1) The non-negativity of $G_{\alpha}(\alpha>0)$ follows immediately from formula (8.3), since the operators $G_{\alpha}^{0}, H_{\alpha},-{\overline{L H_{\alpha}}}^{-1}$ and $\overline{L G_{\alpha}^{0}}$ are all non-negative.

(2) In order to prove inequality (8.6), it suffices to show that

$$
G_{\alpha} 1 \leq \frac{1}{\alpha} \quad \text { on } \bar{\Omega}
$$

since $G_{\alpha}$ is non-negative on $C(\bar{\Omega})$.

First, it follows from the uniqueness property of solutions of the Dirichlet problem (7.1) (Theorem 4.1 with $A:=A-\alpha$ ) that

$$
\alpha G_{\alpha}^{0} 1+H_{\alpha} 1=1+G_{\alpha}^{0} c(x) \quad \text { on } \bar{\Omega} .
$$

Indeed, it suffices to note that the both hand sides of formula (8.8) have the same boundary value 1 and satisfy the same equation: $(\alpha-A) u=\alpha$ in $\Omega$.

By applying the operator $L$ to the both hand sides of formula (8.8), we obtain that

$$
-L H_{\alpha} 1=-L 1-L G_{\alpha}^{0} c+\alpha L G_{\alpha}^{0} 1
$$




$$
\begin{aligned}
& =-\left(\gamma\left(x^{\prime}\right)-\delta\left(x^{\prime}\right) c\left(x^{\prime}\right)\right)-\left(\mu\left(x^{\prime}\right) \frac{\partial}{\partial \mathbf{n}}\left(G_{\alpha}^{0} c\right)+\delta\left(x^{\prime}\right) c\left(x^{\prime}\right)\right)+\alpha L G_{\alpha}^{0} 1 \\
& =-\gamma\left(x^{\prime}\right)-\mu\left(x^{\prime}\right) \frac{\partial}{\partial \mathbf{n}}\left(G_{\alpha}^{0} c\right)+\alpha L G_{\alpha}^{0} 1 \\
& \geq \alpha L G_{\alpha}^{0} 1 \quad \text { on } \partial \Omega
\end{aligned}
$$

since $\left.G_{\alpha}^{0} c\right|_{\partial \Omega}=0$ and $G_{\alpha}^{0} c \leq 0$ on $\bar{\Omega}$. Hence we have, by the non-negativity of $-\overline{L H}_{\alpha}{ }^{-1}$,

$$
-{\overline{L H_{\alpha}}}^{-1}\left(L G_{\alpha}^{0} 1\right) \leq \frac{1}{\alpha} \quad \text { on } \partial \Omega .
$$

By using formula (8.3) with $f:=1$, inequality (8.9) and formula (8.8), we obtain that

$$
\begin{aligned}
G_{\alpha} 1 & =G_{\alpha}^{0} 1+H_{\alpha}\left(-{\overline{L H_{\alpha}}}^{-1}\left(L\left(G_{\alpha}^{0} 1\right)\right)\right) \\
& \leq G_{\alpha}^{0} 1+\frac{1}{\alpha} H_{\alpha} 1=\frac{1}{\alpha}+\frac{1}{\alpha} G_{\alpha}^{0} c \\
& \leq \frac{1}{\alpha} \quad \text { on } \bar{\Omega}
\end{aligned}
$$

since the operators $H_{\alpha}$ and $G_{\alpha}^{0}$ are non-negative.

The proof of Proposition 8.2 is complete.

Corollary 8.1 The operator $\mathfrak{A}$ defined by formula (8.5) is closed in the space $C(\bar{\Omega})$.

Indeed, the closedness of $\mathfrak{A}$ is an immediate consequence of that of the inverse $G_{\alpha}{ }^{-1}=\alpha I-\mathfrak{A}$ (see [45, p. 79, Proposition 3]).

Step (VI): Finally, we prove the following proposition:

Proposition 8.3 The domain $D(\mathfrak{A})$ of the closed operator $\mathfrak{A}$ is dense in the space $C(\bar{\Omega})$.

Proof The proof is divided into two steps.

(1) Before the proof, we need some lemmas on the behavior of the operators $G_{\alpha}^{0}, H_{\alpha}$ and $-\overline{L H}_{\alpha}^{-1}$ as $\alpha \rightarrow+\infty$ (see [34, Lemmas 6.1 and 6.2 and Corollary 6.3], [35, Lemmas 10.24 and 10.25 and Corollary 10.26]):

Lemma 8.4 For all $f \in C(\bar{\Omega})$, we have the assertion

$$
\lim _{\alpha \rightarrow+\infty}\left[\alpha G_{\alpha}^{0} f+H_{\alpha}\left(\left.f\right|_{\partial \Omega}\right)\right]=f \quad \text { in } C(\bar{\Omega}) .
$$

Lemma 8.5 The function

$$
\frac{\partial}{\partial \mathbf{n}}\left(H_{\alpha} 1\right)\left(x^{\prime}\right) \quad \text { for } x^{\prime} \in \partial \Omega,
$$

diverges to $-\infty$ uniformly and monotonically as $\alpha \rightarrow+\infty$.

Corollary 8.2 $\lim _{\alpha \rightarrow+\infty}\left\|-{\overline{L H_{\alpha}}}^{-1}\right\|=0$. 
(2) In view of formula (8.4) and inequality (8.6), it suffices to prove that

$$
\lim _{\alpha \rightarrow+\infty}\left\|\alpha G_{\alpha} f-f\right\|_{C(\bar{\Omega})}=0 \quad \text { for all } f \in C^{2}(\bar{\Omega})
$$

since the space $C^{2}(\bar{\Omega})$ is dense in $C(\bar{\Omega})$.

First, we remark that

$$
\begin{aligned}
\left\|\alpha G_{\alpha} f-f\right\|_{C(\bar{\Omega})}= & \left\|\alpha G_{\alpha}^{0} f-\alpha H_{\alpha}\left({\overline{L H_{\alpha}}}^{-1}\left(\overline{L G_{\alpha}^{0}} f\right)\right)-f\right\|_{C(\bar{\Omega})} \\
\leq & \left\|\alpha G_{\alpha}^{0} f+H_{\alpha}\left(\left.f\right|_{\partial \Omega}\right)-f\right\|_{C(\bar{\Omega})} \\
& +\left\|-\alpha H_{\alpha}\left({\overline{L H_{\alpha}}}^{-1}\left(\overline{L G_{\alpha}^{0}} f\right)\right)-H_{\alpha}\left(\left.f\right|_{\partial \Omega}\right)\right\|_{C(\bar{\Omega})} \\
\leq & \left\|\alpha G_{\alpha}^{0} f+H_{\alpha}\left(\left.f\right|_{\partial \Omega}\right)-f\right\|_{C(\bar{\Omega})} \\
& +\left\|-\alpha{\overline{L H_{\alpha}}}^{-1}\left(\overline{L G_{\alpha}^{0}} f\right)-\left(\left.f\right|_{\partial \Omega}\right)\right\|_{C(\partial \Omega)} .
\end{aligned}
$$

Thus, in view of assertion (8.10) it suffices to show that

$$
\lim _{\alpha \rightarrow+\infty}\left[-\alpha{\overline{L H_{\alpha}}}^{-1}\left(\overline{L G_{\alpha}^{0}} f\right)-\left(\left.f\right|_{\partial \Omega}\right)\right]=0 \quad \text { in } C(\partial \Omega) .
$$

We take a constant $\beta$ such that $0<\beta<\alpha$, and express the function $f \in C^{2}(\bar{\Omega})$ in the form

$$
f=\widetilde{G_{\beta}^{0}} g+H_{\beta} \varphi
$$

where (see formula (7.16) with $u:=f$ and $\alpha:=\beta$ )

$$
\begin{aligned}
\text { - } g & =(\beta-A) f \\
& =(\beta-c(x)) f-\sum_{i, j=1}^{N} a^{i j}(x) \frac{\partial^{2} f}{\partial x_{i} \partial x_{j}}-\sum_{i=1}^{N} b^{i}(x) \frac{\partial f}{\partial x_{i}} \in L^{\infty}(\Omega), \\
\text { - } \varphi & =\left.f\right|_{\partial \Omega} \in C^{2}(\partial \Omega) .
\end{aligned}
$$

Since $f \in C^{2}(\bar{\Omega})$ and $\varphi=\left.f\right|_{\partial \Omega} \in C^{2}(\partial \Omega)$, we find from assertion (7.5), Lemma 7.5 and Remark 7.3 that

$$
\begin{aligned}
& L H_{\alpha} \varphi \in B^{1-1 / p, p}(\partial \Omega) \subset C(\partial \Omega), \\
& L f=L\left(\widetilde{G_{\beta}^{0}} g+H_{\beta} \varphi\right)=\widetilde{L G_{\beta}^{0}} g+L H_{\beta} \varphi \in C(\partial \Omega) .
\end{aligned}
$$

Moreover, by using the resolvent equation (7.7) with $f:=g \in L^{\infty}(\Omega)$ (see Remark 7.1) and the equation (7.10) with $\varphi \in C^{2}(\partial \Omega)$, we obtain from formula (8.13) that

$$
G_{\alpha}^{0} f=G_{\alpha}^{0}\left(\widetilde{G_{\beta}^{0}} g\right)+G_{\alpha}^{0}\left(H_{\beta} \varphi\right)=\frac{1}{\alpha-\beta}\left(\widetilde{G_{\beta}^{0}} g-\widetilde{G_{\alpha}^{0}} g+H_{\beta} \varphi-H_{\alpha} \varphi\right) .
$$

By combining formulas (8.15) and (8.14b), we have the inequality

$$
\begin{aligned}
& \left\|-\alpha{\overline{L H_{\alpha}}}^{-1}\left(\overline{L G_{\alpha}^{0}} f\right)-\left(\left.f\right|_{\partial \Omega}\right)\right\|_{C(\partial \Omega)} \\
= & \left\|\frac{\alpha}{\alpha-\beta}\left(-{\overline{L H_{\alpha}}}^{-1}\right)\left(\widetilde{L G_{\beta}^{0}} g-\widetilde{L G_{\alpha}^{0}} g+L H_{\beta} \varphi-L H_{\alpha} \varphi\right)-\varphi\right\|_{C(\partial \Omega)}
\end{aligned}
$$




$$
\begin{aligned}
& =\left\|\frac{\alpha}{\alpha-\beta}\left(-{\overline{L H_{\alpha}}}^{-1}\right)\left(\widetilde{L G_{\beta}^{0}} g-\widetilde{L G_{\alpha}^{0}} g+L H_{\beta} \varphi\right)+\frac{\alpha}{\alpha-\beta} \varphi-\varphi\right\|_{C(\partial \Omega)} \\
& =\left\|\frac{\alpha}{\alpha-\beta}\left(-{\overline{L H_{\alpha}}}^{-1}\right)\left(L f-\widetilde{L G_{\alpha}^{0}} g\right)+\frac{\beta}{\alpha-\beta} \varphi\right\|_{C(\partial \Omega)} \\
& \leq \frac{\alpha}{\alpha-\beta}\left\|-{\overline{L H_{\alpha}}}^{-1}\right\| \cdot\|L f\|_{C(\partial \Omega)} \\
& +\frac{\alpha}{\alpha-\beta}\left\|-{\overline{L H_{\alpha}}}^{-1}\right\| \cdot\left\|\overline{L G_{\alpha}^{0}}\right\| \cdot\|g\|_{L^{\infty}(\Omega)}+\frac{\beta}{\alpha-\beta}\|\varphi\|_{C(\partial \Omega)} .
\end{aligned}
$$

However, by Corollary 8.2 it follows that the first term on the last inequality (8.16) converges to zero as $\alpha \rightarrow+\infty$.

For the second term, by using the resolvent equation (7.7) with $f:=1$ and the non-negativity of $G_{\beta}^{0}$ and $\widetilde{L G_{\alpha}^{0}}$ we obtain that

$$
\begin{aligned}
\left\|\overline{L G_{\alpha}^{0}}\right\| & =\left\|L\left(G_{\beta}^{0} 1\right)-(\alpha-\beta) \overline{L G_{\alpha}^{0}}\left(G_{\beta}^{0} 1\right)\right\|_{C(\partial \Omega)} \\
& \leq\left\|L\left(G_{\beta}^{0} 1\right)\right\|_{C(\partial \Omega)}=\left\|\overline{L G_{\beta}^{0}}\right\| \quad \text { for all } \alpha>0 .
\end{aligned}
$$

Hence, by Corollary 8.2 it follows that the second term on the last inequality (8.16) also converges to zero as $\alpha \rightarrow+\infty$. It is clear that the third term on the last inequality (8.16) converges to zero as $\alpha \rightarrow+\infty$. This completes the proof of assertion (8.12) and hence of assertion (8.11).

The proof of Proposition 8.3 is now complete.

Step (VII): By combining Propositions 8.1, 8.2 and 8.3 and Corollary 8.1, we obtain that the closed operator $\mathfrak{A}$, defined by formula (8.5), satisfies conditions (a) through (d) in Theorem 3.2. Therefore, it follows from an application of the same theorem that the closed operator $\mathfrak{A}$ is the infinitesimal generator of some Feller semigroup on the state space $\bar{\Omega}$.

Now the proof of Theorem 1.1 is complete.

\section{Proof of Remark 7.2}

Finally, we prove that the domain

$$
D(\bar{A})=\left\{u \in W^{2, p}(\Omega): A u \in C(\bar{\Omega})\right\}
$$

is independent of $p$, for $N<p<\infty$.

The proof is divided into three steps.

Step 1: First, we consider the following non-homogeneous Dirichlet boundary value problem

$$
\begin{cases}A u=f & \text { in } \Omega, \\ \gamma_{0} u=\varphi & \text { on } \partial \Omega .\end{cases}
$$

Our proof is based on the following regularity theorem due to Vitanza [42, Theorem 2.2] (see also [13, Theorem 4.2]): 
Theorem 9.1 (the regularity theorem) Let $1<p<\infty$. If a function $u \in W^{2, q}(\Omega)$ for $1<q<p<\infty$ is a solution of the Dirichlet problem (4.1) with $f \in L^{p}(\Omega)$ and $\varphi \in B^{2-1 / p, p}(\partial \Omega)$, then it follows that $u \in W^{2, p}(\Omega)$.

Moreover, we have the global a priori estimate

$$
\|u\|_{W^{2, p}(\Omega)} \leq C\left(\|u\|_{L^{p}(\Omega)}+\|f\|_{L^{p}(\Omega)}+\|\varphi\|_{B^{2-1 / p, p}(\partial \Omega)}\right),
$$

with a constant $C>0$.

Step 2: Now we let

$$
\mathcal{E}_{p}:=\left\{u \in W^{2, p}(\Omega): A u \in C(\bar{\Omega})\right\} .
$$

In order to prove Remark 7.2 , it suffices to show that

$$
\mathcal{E}_{p_{1}}=\mathcal{E}_{p_{2}} \quad \text { for } N<p_{1}<p_{2}<\infty .
$$

First, it follows that

$$
\mathcal{E}_{p_{2}} \subset \mathcal{E}_{p_{1}}
$$

since we have the inclusion

$$
L^{p_{2}}(\Omega) \subset L^{p_{1}}(\Omega) \text { for } p_{2}>p_{1} .
$$

Step 3: Conversely, let $v$ be an arbitrary element of $\mathcal{E}_{p_{1}}$ :

$$
v \in W^{2, p_{1}}(\Omega), \quad A v \in C(\bar{\Omega}) .
$$

Then, since we have the assertions

$$
v, A v \in C(\bar{\Omega}) \subset L^{p_{2}}(\Omega),
$$

it follows from an application of Theorem 9.1 with $p:=p_{2}$ and $\varphi:=0$ that

$$
G_{\alpha}^{0}((\alpha-A) v) \in W^{2, p_{2}}(\Omega),
$$

where $G_{\alpha}^{0}$ is the Green operator introduced in part (i) of Theorem 7.1.

Moreover, we can find a sequence $\left\{\varphi_{j}\right\}$ in $C^{2}(\partial \Omega)$ such that

$$
\left.\varphi_{j} \longrightarrow v\right|_{\partial \Omega} \quad \text { in } C(\partial \Omega) \text {. }
$$

Then we have the assertions

$$
\begin{cases}H_{\alpha} \varphi_{j} \in W^{2, p_{2}}(\Omega), & \\ H_{\alpha} \varphi_{j} \longrightarrow H_{\alpha}\left(\left.v\right|_{\partial \Omega}\right) & \text { in } C(\bar{\Omega}), \\ A\left(H_{\alpha} \varphi_{j}\right)=\alpha H_{\alpha} \varphi_{j} \longrightarrow \alpha H_{\alpha}\left(\left.v\right|_{\partial \Omega}\right) & \text { in } C(\bar{\Omega}),\end{cases}
$$

where $H_{\alpha}$ is the harmonic operator introduced in part (ii) of Theorem 7.1.

However, it follows from an application of Lemma 7.1 wit $p:=p_{2}$ that the operator

$$
\begin{gathered}
\bar{A}_{p_{2}}: \mathcal{E}_{p_{2}} \longrightarrow C(\bar{\Omega}) \\
u \longmapsto A u
\end{gathered}
$$


is closed. Hence we have, by assertions (9.2),

$$
\begin{aligned}
& H_{\alpha}\left(\left.v\right|_{\partial \Omega}\right) \in D\left(\bar{A}_{p_{2}}\right)=\mathcal{E}_{p_{2}} \subset W^{2, p_{2}}(\Omega), \\
& \bar{A} H_{\alpha}\left(\left.v\right|_{\partial \Omega}\right)=\alpha H_{\alpha}\left(\left.v\right|_{\partial \Omega}\right) .
\end{aligned}
$$

Therefore, by applying Corollary 7.1 with $\bar{A}:=\bar{A}_{p_{2}}$ and $u:=v$ we obtain from assertions (9.1) and (9.3a) that

$$
v=G_{\alpha}^{0}\left(\left(\alpha I-\bar{A}_{p_{2}}\right) v\right)+H_{\alpha}\left(\left.v\right|_{\partial \Omega}\right)=G_{\alpha}^{0}((\alpha-A) v)+H_{\alpha}\left(\left.v\right|_{\partial \Omega}\right) \in W^{2, p_{2}}(\Omega) .
$$

This implies that

$$
v \in \mathcal{E}_{p_{2}} .
$$

The proof of Remark 7.2 is complete.

\section{Concluding Remarks}

In the previous paper [34], we assumed the additional condition (H.2) on the boundary condition $L_{\nu}$ defined by formula (1.5). The main purpose of the present paper is how to drop this additional condition (H.2). In the proof of Theorems 1.1 and 1.2 we re-work and expand in different sprit the material of the previous paper [34] under condition (H.1), even though there is a lot of overlap in the contents of the present paper and those of [34]. This makes the present paper fairly self-contained.

\begin{tabular}{|c|c|c|}
\hline $\begin{array}{l}\text { Elliptic boundary } \\
\text { value Problems }\end{array}$ & $\begin{array}{l}\text { Classical } \\
\text { Schauder theory }\end{array}$ & $\begin{array}{c}\text { Calderón-Zygmund } \\
\text { theory }\end{array}$ \\
\hline $\begin{array}{l}\text { - Dirichlet case } \\
\text { - Feller semigroup } \\
\text { with sticking term }\end{array}$ & $\begin{array}{l}{[8, \text { Théorème XV] }} \\
{[8, \text { Théorème XVI }]}\end{array}$ & $\begin{array}{l}{[33, \text { Theorem 1.2] }} \\
{[33, \text { Theorem 1.1] }}\end{array}$ \\
\hline $\begin{array}{l}\text { - Oblique derivative } \\
\text { case } \\
\text { - Feller semigroup } \\
\text { with sticking term }\end{array}$ & $\begin{array}{c}{[8, \text { Théorème XVIII] }} \\
\text { (under }(\mathrm{H} .1)) \\
{[8, \text { Théorème XIX] }} \\
\quad(\text { under }(\text { H.1) })\end{array}$ & $\begin{array}{c}{[34, \text { Theorem 1.2] }} \\
\text { (under (H.1) and (H.2)) } \\
\\
{[34, \text { Theorem 1.1] }} \\
\text { (under (H.1) and (H.2)) }\end{array}$ \\
\hline
\end{tabular}

Finally, for general results on generation theorems for Feller semigroups we give the following overview of the classical Schauder theory versus the CalderónZygmund theory of singular integrals:

Table 10.1 An overview of the classical Schauder theory versus the Calderón-Zygmund theory of singular integrals for Feller semigroups

Acknowledgements I am grateful to the referee for valuable suggestions and for informing me of an additional reference, which improved the presentation of this paper. 


\section{References}

1. Acquistapace, P., On BMO regularity for linear elliptic systems, Ann. Mat. Pura Appl. 161, 231-269 (1992).

2. Adams, R. A. and Fournier, J. J. F., Sobolev spaces, second edition, Pure and Applied Mathematics, Vol. 140, Elsevier/Academic Press, Amsterdam, 2003.

3. Agmon, S., Douglis, A. and Nirenberg, L., Estimates near the boundary for solutions of elliptic partial differential equations satisfying general boundary conditions I, Comm. Pure Appl. Math. 12, 623-727 (1959).

4. Amann, H., Nonlinear elliptic equations with nonlinear boundary conditions, In: New developments in differential equations, W. Eckhaus (ed.), North-Holland Mathematics Studies, Vol. 21 (North-Holland, Amsterdam New York Oxford), pp. 43-63 (1976).

5. Bergh, J. and Löfström, J., Interpolation spaces, an introduction, Springer-Verlag, Berlin Heidelberg New York, 1976.

6. Bony, J.-M., Principe du maximum dans les espaces de Sobolev, C. R. Acad. Sc. Paris Sér. A-B 265, A333-A336 (1967).

7. Bony, J.-M., Problème de Dirichlet et semi-groupe fellérien associés à un opérateur intégrodifférentiel, C. R. Acad. Sc. Paris Sér. A-B 265, A361-A364 (1967).

8. Bony, J.-M., Courrège, P. et Priouret, P., Semi-groupes de Feller sur une variété à bord compacte et problèmes aux limites intégro-différentiels du second ordre donnant lieu au principe du maximum, Ann. Inst. Fourier (Grenoble) 18, 369-521 (1968).

9. Boutet de Monvel, L., Boundary problems for pseudo-differential operators, Acta Math. 126, 11-51 (1971)

10. Bramanti, M., Commutators of integral operators with positive kernels, Matematiche (Catania) 49, 149-168 (1994).

11. Calderón, A. P. and Zygmund, A., On the existence of certain singular integrals, Acta. Math. 88, 85-139 (1952).

12. Chiarenza, F., Frasca, M. and Longo, P., Interior $W^{2, p}$ estimates for nondivergence elliptic equations with discontinuous coefficients, Ricerche Mat. 60, 149-168 (1991).

13. Chiarenza, F., Frasca, M. and Longo, P., $W^{2, p}$-solvability of the Dirichlet problem for nondivergence elliptic equations with VMO coefficients, Trans. Amer. Math. Soc. 336, 841-853 (1993).

14. Di Fazio, G. and Palagachev, D. K., Oblique derivative problem for elliptic equations in non-divergence form with VMO coefficients. Comment. Math. Univ. Carolinae 37, 537556 (1996).

15. Dynkin, E. B., Foundations of the theory of Markov processes, Fizmatgiz, Moscow, 1959 (Russian); English translation: Pergamon Press, Oxford London New York Paris, 1960; German translation: Springer-Verlag, Berlin Göttingen Heidelberg, 1961; French translation: Dunod, Paris, 1963.

16. Dynkin, E. B., Markov processes I, II, Springer-Verlag, Berlin Göttingen Heidelberg, 1965.

17. Fiorenza, R., Sui problemi di derivata obliqua per le equazioni ellittiche. Ricerche Mat. 8 (1959), 83-110.

18. Garnett, J. B., Bounded analytic functions. Academic Press, New York London Toronto Sydney San Francisco, 1981.

19. Gilbarg, D. and Trudinger, N. S., Elliptic partial differential equations of second order, 1998 edition, Springer-Verlag New York Berlin Heidelberg Tokyo, 1998.

20. Gohberg, I. G. and Krĕn, M. G., The basic propositions on defect numbers, root numbers and indices of linear operators (Russian). Uspehi Mat. Nauk. 12, 43-118 (1957). English translation: Amer. Math. Soc. Transl. (2) 13, 185-264 (1960).

21. John, F. and Nirenberg, L., On functions of bounded mean oscillation, Comm. Pure and Appl. Math. 14, 415-426 (1961).

22. Lieberman, G. M., Local estimates for subsolutions and supersolutions of oblique derivative problems for general second order elliptic equations, Trans. Amer. Math. Soc. 304, 343353 (1987).

23. Malý, J. and Ziemer, W. P., Fine regularity of solutions of elliptic partial differential equations, American Mathematical Society, Providence, Rhode Island, 1997.

24. Maugeri, A. and Palagachev, D. K., Boundary value problem with an oblique derivative for uniformly elliptic operators with discontinuous coefficients, Forum Math. 10, 393-405 (1998).

25. Maugeri, A., Palagachev, D. K. and Softova, L. G., Elliptic and parabolic equations with discontinuous coefficients, Mathematical Research, 109, Wiley-VCH, Berlin, 2000. 
26. Meyers, N., An $L^{p}$-estimate for the gradient of solutions of second order elliptic divergence equations, Ann. Scuola Norm. Sup. Pisa 17, 189-206 (1963).

27. Miranda, C., Sulle equazioni ellittiche del secondo ordine di tipo non variazionale a coefficienti discontinui, Ann. Mat. Pura Appl. 63, 353-386 (1963).

28. Sarason, D., Functions of vanishing mean oscillation, Trans. Amer. Math. Soc. 207, 391405 (1975).

29. Sato, K. and Ueno, T., Multi-dimensional diffusion and the Markov process on the boundary, J. Math. Kyoto Univ. 4, 529-605 (1965).

30. Schechter, M., Principles of functional analysis, second edition. Graduate Studies in Mathematics, Vol. 36, American Mathematical Society, Providence, Rhode Island, 2002.

31. Stein, E. M., Harmonic analysis: real-variable methods, orthogonality, and oscillatory integrals, Princeton Univ. Press, Princeton, New Jersey, 1993.

32. Taira, K., Diffusion processes and partial differential equations, Academic Press, San Diego New York London Tokyo, 1988.

33. Taira, K., On the existence of Feller semigroups with discontinuous coefficients, Acta Math. Sinica (English Series) 22, 595-606 (2006).

34. Taira, K., On the existence of Feller semigroups with discontinuous coefficients II, Acta Math. Sinica (English Series) 25, 715-740 (2009).

35. Taira, K., Semigroups, boundary value problems and Markov processes, second edition, Springer Monographs in Mathematics, Springer-Verlag, Berlin Heidelberg New York, 2014.

36. Taira, K., Dirichlet problems with discontinuous coefficients and Feller semigroups, Rend. Circ. Mat. Palermo (2) Advance online publication. Doi:10.1007/s12215-019-00404-5

37. Taira, K., Ventcel' boundary value problems for elliptic Waldenfels operators, Boll. Unione Mat. Ital.

Advance online publication. Doi:10.1007/s40574-019-00214-8

38. Talenti, G., Equazioni lineari ellittiche in due variabili, Matematiche (Catania) 21, 339376 (1966).

39. Torchinsky, A., Real-variable methods in harmonic analysis, Dover Publications Inc., Mineola, New York, 2004

40. Triebel, H., Theory of function spaces, Birkhäuser, Basel Boston Stuttgart, 1983.

41. Troianiello, G. M., Elliptic differential equations and obstacle problems, The University Series in Mathematics, Plenum Press, New York, 1987.

42. Vitanza, C., $W^{2, p}$-regularity for a class of elliptic second order equations with discontinuous coefficients, Le Matematiche 47, 177-186 (1992).

43. von Waldenfels, W., Positive Halbgruppen auf einem $n$-dimensionalen Torus, Arch. Math. 15, 191-203 (1964).

44. Wentzell (Ventcel'), A. D., On boundary conditions for multidimensional diffusion processes (Russian), Teoriya Veroyat. i ee Primen. 4, 172-185 (1959). English translation in Theory Prob. and its Appl. 4, 164-177 (1959).

45. Yosida, K., Functional analysis, reprint of the sixth (1980) edition, Classics in Mathematics, Springer-Verlag, Berlin Heidelberg New York, 1995. 